\title{
Unravelling a novel sol-gel transition mechanism in polymer self-assemblies: An order-order transition based on specific molecular interactions between hydrophilic and hydrophobic polymer blocks
}

* This contribution is dedicated to the late Françoise Winnik

Lukas Hahn' ${ }^{1}$, Theresa Zorn' ${ }^{2}$, Josef Kehrrein ${ }^{3}$, Tobias Kielholz ${ }^{4}$, Benedikt Sochor ${ }^{5}$, Ekaterina S. Lisitsyna ${ }^{6}$, Nikita A. Durandin 6 , Timo Laaksonen, ${ }^{6,7}$ Vladimir Aseyev $^{8}$, Christoph Sotriffer ${ }^{3}$, Maike Windbergs ${ }^{4}$, Ann-Christin Pöppler, ${ }^{2, *}$ and Robert Luxenhofer ${ }^{1,8, *}$

${ }^{1}$ Functional Polymer Materials, Chair for Advanced Materials Synthesis, Institute for Functional Materials and Biofabrication, Department of Chemistry and Pharmacy, Julius-Maximilians-University Würzburg, Röntgenring 11, 97070 Würzburg, Germany

${ }^{2}$ Institute of Organic Chemistry, Department of Chemistry and Pharmacy, Julius-Maximilians-University Würzburg, Am Hubland, 97074 Würzburg, Germany

${ }^{3}$ Institute of Pharmacy and Food Chemistry, Department of Chemistry and Pharmacy, JuliusMaximilians-University Würzburg, Am Hubland, 97074 Würzburg, Germany

${ }^{4}$ Institute of Pharmaceutical Technology and Buchmann Institute for Molecular Life Sciences, Goethe University Frankfurt, Max-von-Laue-Str. 15, 60438 Frankfurt am Main, Germany

${ }^{5}$ Chair for X-Ray Microscopy, Julius-Maximilians-University Würzburg, Josef-Martin-Weg 63, 97074 Würzburg, Germany

${ }^{6}$ Faculty of Engineering and Natural Science, Tampere University, Korkeakoulunkatu 8, 33720 Tampere, Finland

${ }^{7}$ Division of Pharmaceutical Biosciences, Faculty of Pharmacy, University of Helsinki, Viikinkaari $5 \mathrm{E}$, 00014 Helsinki, Finland

${ }^{8}$ Soft Matter Chemistry, Department of Chemistry, and Helsinki Institute of Sustainability Science, Faculty of Science, Helsinki University, 00014 Helsinki, Finland

*correspondence to: robert.luxenhofer@helsinki.fi, ann-christin.poeppler@uni-wuerzburg.de

Keywords: Inverse gelation, poly(2-oxazoline), poly(2-oxazine), aromatic moieties, worm-to-sphere transition 


\section{Abstract}

Thermoresponsive hydrogel formation upon cooling in aqueous media is rarely described for synthetic polymers in the literature. However, if the sol/gel transition occurs in the physiologically relevant range $\left(0-40^{\circ} \mathrm{C}\right)$, there are many possible applications in areas such as drug delivery and biofabrication. Here, a novel inverse thermoresponsive polymer self-assembly mechanism based on a poly(2oxazoline)/poly(2-oxazine) amphiphile is investigated in detail. Dynamic light scattering and smallangle X-ray scattering confirmed worm-to-spheres transition upon heating on the nanoscale level while wide-angle $\mathrm{X}$-ray scattering indicated a more uniform ordering of the macromolecular chains on the scale of 4-7 $\AA$. NMR spectroscopy shows reduced mobility of various polymer segments in the hydrogel state, especially in the hydrophobic aromatic region. More importantly, it also reveals close proximity of the phenyl ring of the hydrophobic block with hydrophilic repeat units confirmed by solution and solid-state NMR investigations, suggesting interactions between the two. This unusual interaction is corroborated in silico by molecular dynamics modeling. We propose a novel order-order transition based on unexpected and previously not described interactions between the hydrophilic and the hydrophobic repeating units, which opens new avenues to control and design macromolecular selfassembly. 


\section{Introduction}

Thermoresponsive phase separation of polymer solutions upon heating is an entropic effect and widely discussed in literature. ${ }^{1}$ Polymers displaying a lower critical solution temperature (LCST) are structurally diverse and can be found in the families of poly(ether)s, poly(acrylamide)s, poly(2oxazoline)s and many others. In contrast, examples of UCST (upper critical solution temperature) type phase separation are more rarely found, especially for UCST in purely aqueous media, and have only recently been described. ${ }^{2,3}$ However, such systems offer a variety of different applications as smart biomaterials if the transition takes place at or around physiological temperatures and in physiological media. In some cases, physical hydrogel formation (sol-gel transition) can be observed instead of precipitation (sol/coil-globule transition), due to changing aggregation patterns. Block copolymers with thermogelling properties are well-known in literature, ${ }^{4,5}$ with Pluronic ${ }^{\circledR}$ F127 being arguably the most prominent example. ${ }^{6}$ In many cases, polymer micelles form dense colloidal packings, which lead to gelation, upon increasing the temperature. ${ }^{7}$

The polymer families of poly(2-substituted-2-oxazoline)s (POx) and - to a lesser extent - poly(2substituted-5,6-dihydro-4H-1,3-oxazines), in short poly(2-oxazine)s (POzi), have been described as smart biomaterials owing to their excellent cyto- and biocompatibility and chemical versatility. ${ }^{8-11}$ In general, POx and POzi homopolymers can exhibit LCST behavior, in particular when bearing $\mathrm{C}_{3}$ alkyl sidechains. By varying the degree of polymerization and polymer composition, the LCST can be tuned as described by Winnik and co-workers. By using telechelic hydrophobically modified poly(2-alkyl-2oxazolines) in water, different aggregates were obtained at different temperatures. ${ }^{12-15} \mathrm{~A}$ detailed phase diagram of such aggregates depending on concentration and temperature was determined confirming the presence of star micelles, microgels, mesoglobules and microfibers. Overall, the literature is rich with respect to thermoresponsive POx and to a lesser extent of poly(2-oxazine) based polymers. However, the first thermogelling hydrogel comprising exclusively POx/POzi was described only fairly recently. ${ }^{4} \mathrm{~A}$ diblock copolymer exhibiting a hydrophilic pMeOx block and a thermoresponsive poly(2-n-proply-2-oxazine) (pnPrOzi) block undergoes heating induced physical 
gelation by forming a sponge-like network formed from polymer vesicles. Subsequently, also thermogelling polymers solely based on POx have been described. ${ }^{16,17}$ These thermogelling materials rely on a thermally triggered disorder-order transition. In contrast, Armes and co-workers described an interesting and unusual heating induced worm-to-sphere order-order transition. ${ }^{18}$ By introducing di(ethylene glycol) methyl ether methacrylate (DEGMA) moieties into the core-forming block of the polymer, the authors were able to increase and tune the critical gelation temperature. ${ }^{19}$ In 2017 , Penfold et al. introduced a system bearing $\mathrm{pH}$-sensitive end groups that triggered a vesicle-to-worm transition in combination with thermosensitive worm-to-sphere transitions. ${ }^{20}$ More recently, a thermoresponsive poly(N-(2-hydroxypropyl)methacrylamide)-poly(2-hydroxypropyl)methacrylate) diblock copolymer was described, which formed spheres $\left(4^{\circ} \mathrm{C}\right.$, weakly turbid free flowing fluid), worms $\left(22^{\circ} \mathrm{C}\right.$, turbid free standing gel) or vesicles $\left(50^{\circ} \mathrm{C}\right.$, milky-white free flowing dispersion) in aqueous solution. ${ }^{21}$

Very recently, the first cooling induced and reversible sphere-to-worm transition in aqueous solutions of a novel ABA-type block copolymer amphiphile poly(2-methyl-2-oxazoline)-b-poly(2-phenyl-2oxazine)- $b$-poly(2-methyl-2-oxazoline) (pMeOx- $b$-pPheOzi- $b$-pMeOx $=$ A-pPheOzi-A) was described (Figure 1). ${ }^{22}$ Apart from the macroscopic change, the unexpected order-order transition was observed via cryo transmission electron microscopy (cryoTEM) analysis. At $5{ }^{\circ} \mathrm{C}$, the polymer exhibited long interconnected worm-like aggregates, which transformed into small, spherical polymer micelles upon heating. The temperature, where the viscosity of the system decreases several orders of magnitude, is $32{ }^{\circ} \mathrm{C}$. We hypothesize that a critical number of worms transforms into spheres at this temperature. Here, using a variety of state-of-the-art analytic tools complemented by molecular modeling, we aim to elucidate the molecular origins of this novel gelation mechanism in detail. We found that the novel transition in self-assembly is based on a unique interaction between the hydrophilic pMeOx blocks and the aromatic moieties in the hydrophobic pPheOzi block, leading to a compaction of the former onto the latter, which concomitantly leads to the sphere-to-worm morphology transition as the packing parameter increases. 


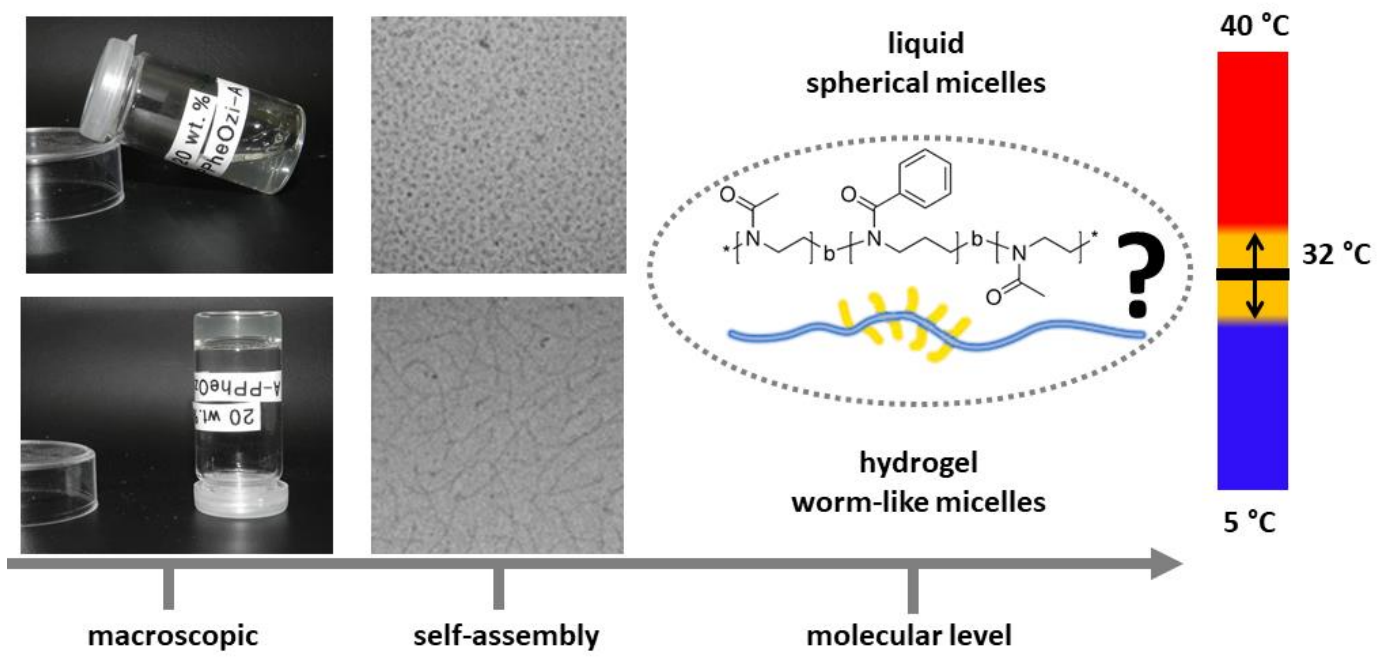

Figure 1| Summary of the study. The polymer amphiphile poly(2-methyl-2-oxazoline)-b-poly(2-phenyl-2oxazine)-b-poly(2-methyl-2-oxazoline) (pMeOx-b-pPheOzi- $b$-pMeOx = A-PPheOzi-A = A-B-A) showed unique inverse thermogelling properties. ${ }^{22}$ In the liquid state the polymer self-assembled into spherical micelles. By cooling an order-order transition into worm-like micelles was observed. In this study a detailed elucidation of the order-order transition is presented with the focus on polymer interactions on molecular level. The color code is used alongside this study to highlight the samples macroscopic state. 


\section{Materials and Methods}

All chemicals and reagents were used from Sigma-Aldrich (Steinheim, Germany) or TCl-chemicals (Eschborn, Germany) and were used as received unless otherwise mentioned. The polymer MepMeOx- $b$-pPheOzi- $b$-pMeOx (= A-pPheOzi-A) was prepared and used as described previously. ${ }^{22}$

Dynamic light scattering experiments (DLS) were performed using an ALV CGS-3 multi detection goniometry-system (Langen, Germany) equipped with a He-Ne-laser (632.8 nm) and 8 optical avalanche photodiodes-detector with an angular detector spacing of $16^{\circ}$ (correlation time $45 \mathrm{~s}, 3$ runs). Scattering angles between 20 and $147^{\circ}$ were measured in 4 angle sets ( $4 \times 8$ detectors) and a $5^{\circ}$ angle interval for each detector at $15{ }^{\circ} \mathrm{C}$ and $40{ }^{\circ} \mathrm{C}$. Prior to each measurement, samples were filtered in dust-free cuvettes using Millex-LG $0.2 \mu \mathrm{m}$ filters under laminar flow. The polymer concentration was $0.1 \mathrm{~g} / \mathrm{L}\left(2 \mathrm{mM}\right.$ aqueous $\left.\mathrm{NaNO}_{3}\right)$. All samples were stored for $24 \mathrm{~h}$ at the measurement temperature. The decay of the electric field-time autocorrelation function (ACF) was fitted using triexponential fit functions (equation 1) like described previously. ${ }^{23}$

$$
g_{1}(t)=a_{1} \cdot e^{\left(-\frac{t}{\tau_{1}}\right)}+a_{2} \cdot e^{\left(-\frac{t}{\tau_{2}}\right)}+a_{3} \cdot e^{\left(-\frac{t}{\tau_{3}}\right)}
$$

With the amplitude $a_{i}$, decay times $\tau_{i}=\frac{1}{q^{2} \cdot D_{i}}$ and the absolute value of the scattering vector $q$. In the case of polydispersity, the translational diffusion coefficient $D$ is obtained by extrapolation to zero angle and in the limit of high dilution given by

$$
\langle D\rangle_{z}^{-1}=\frac{\sum_{i} a_{i}}{\sum_{i} a_{i} \cdot D_{i}^{-1}}
$$

Using the Stokes-Einstein equation the hydrodynamic radius $R_{h}$ is obtained by

$$
R_{h}=\frac{k_{B} \cdot T}{6 \cdot \pi \cdot \eta \cdot D}
$$

with $k_{B}$ being the Boltzmann constant, $\eta$ is the viscosity of the solvent and $T$ the temperature $\left(15^{\circ} \mathrm{C}\right.$ or $40^{\circ} \mathrm{C}$. 
Small- and wide-angle X-ray scattering experiments (SAXS, WAXS) were carried out using an in-house setup, which was built by Fraunhofer EZRT (Fürth, Germany). It consists of a MicroMax-007 HF X-ray source (Rigaku, Japan) and a Eiger R 1M detector unit (Dectris, Switzerland). The sample-detector distance can be varied between $5 \mathrm{~cm}$ and $3.5 \mathrm{~m}$, which corresponds to possible Q-values between 0.005 and $5 \AA^{-1}$. The complete setup is operated in a vacuum below $0.1 \mathrm{mbar}$ to reduce air scattering. The sample solutions were placed in quartz capillaries (inner diameter: $1 \mathrm{~mm}$, wall thickness: $10 \mu \mathrm{m}$ ) (Hampton Research, Aliso Viejo, California), which were positioned perpendicularly to the X-ray beam. The presented experiments were done at sample-detector distances of $57 \mathrm{~mm}, 565 \mathrm{~mm}$ and $1560 \mathrm{~mm}$ with an integration time of 15 minutes for the shortest distance and 240 minutes for the two longer configurations. All distances were calibrated using a silver behenate standard sample. For each sample, data was acquired for different temperatures between $5-50^{\circ} \mathrm{C}$. To achieve thermal equilibrium, the sample (10 wt.\% aqueous solution) was kept at the desired temperature for 15 minutes prior to each measurement. The SAXS data, which was obtained at the two largest distances, was calibrated in terms of absolute intensities using glassy carbon as a secondary calibration standard. ${ }^{24,25}$ The scattering curves of the hydrogels were obtained by azimuthal integration taking the samples thickness, X-ray transmission, detector accuracy, setup geometry and solvent scattering into account following the standard procedures described in literature. ${ }^{26}$

\section{Temperature dependent nuclear magnetic resonance experiments in solution}

All experiments were performed at a Bruker Avance III HD 600 spectrometer (Karlsruhe, Germany) operating at $600.4 \mathrm{MHz}$ equipped with a BBFO $5 \mathrm{~mm}$ probe using a BCU-02 (Bruker) temperature control unit. $\mathrm{D}_{2} \mathrm{O}$ was obtained from Deutero $\mathrm{GmbH}$ (Kastellaun, Germany). ${ }^{1} \mathrm{H}$ NMR experiments of a 20 wt.\% A-pPheOzi ${ }_{15}-\mathrm{A}$ sample in $\mathrm{D}_{2} \mathrm{O}$ were acquired with a $30^{\circ}$ flip angle and 8 or 16 scans without sample spinning. A series of variable temperature experiments was performed in the range from $2{ }^{\circ} \mathrm{C}$ to $39^{\circ} \mathrm{C}$ in a step size of 3 to $5^{\circ} \mathrm{C}$. The sample was kept for 10 minutes at the desired temperature prior to each measurement. Temperature calibration was done using $4 \% \mathrm{MeOH}$ in $\mathrm{MeOD}$ and $80 \%$ ethylene glycol in DMSO- $\mathrm{d}_{6}$. All recorded spectra were referenced using the temperature dependent HDO signal. 
For quantitative characterization of the temperature induced phase transition the fraction $p$ was calculated with the integrals $I(T)$ and $I\left(T_{0}\right)$ at the respective temperatures $T$ and $T_{0}$ using the following equation: ${ }^{27}$

$$
p=1-\frac{I(T)}{I\left(T_{0}\right) * \frac{T_{0}}{T}}
$$

The highest signal intensity was measured at $39{ }^{\circ} \mathrm{C}\left(T_{0}\right)$. Reductions of signal intensities caused by inhibited molecular mobility due to the transition of spherical to worm-like micelles could be quantified by $p<0$. 2D ${ }^{1} \mathrm{H}-{ }^{1} \mathrm{H}$ NOESY NMR experiments at $7{ }^{\circ} \mathrm{C}$ and $40{ }^{\circ} \mathrm{C}$ were recorded using the noesygpphpp pulse sequence (scans: $32, t_{1}$ increments: 256 , relaxation delay: $2.5 \mathrm{~s}$ ). To ensure discrimination between cross-relaxation and spin-diffusion different mixing times ( $40 \mu \mathrm{s}, 60 \mu \mathrm{s}, 80 \mu \mathrm{s}$, $150 \mu \mathrm{s}$ and $250 \mu \mathrm{s})$ were used. For better visualization of the aromatic region, phase and baseline correction of $2 \mathrm{D}$ data was confined to the range of $6-8 \mathrm{ppm}$ and TDeff was set to 2867 in the f2 dimension. Additionally, 1D slices of relevant aromatic regions were extracted. ${ }^{1} \mathrm{H}$ spin-lattice relaxation times $\mathrm{T} 1$ were measured with the inversion recovery pulse sequence t1ir at $7^{\circ} \mathrm{C}$ and $40^{\circ} \mathrm{C}$. For measuring the T1 relaxation times of HDO a variable delay from 1-50 s in 12 steps was used with a relaxation delay of $50.0 \mathrm{~s}$. T1 relaxation times of polymer signals were recorded with a variable delay from $0.001-5.0 \mathrm{~s}$ in 16 steps and a relaxation delay of $5.0 \mathrm{~s}$. The normalized decay curves for different polymer segments and the HDO signal were fitted using monoexponential fit functions.

\section{Solid-state nuclear magnetic resonance (sSNMR)}

The solid-state NMR measurements were performed using a $4 \mathrm{~mm}$ double-channel Bruker probe at $9.4 \mathrm{~T}$ using between 3 and $5.3 \mathrm{kHz}$ MAS. The hydrogel sample was cooled to $273 \mathrm{~K}$ prior to measurement. Due to small amounts of frictional heating through MAS, the actual sample temperature is slightly higher. For the ${ }^{13} \mathrm{C} C P$ MAS experiment, a $2 \mathrm{~ms}$ ramp (50 to $100 \%$ ) on the ${ }^{1} \mathrm{H}$ channel was used during the $\mathrm{CP}$ contact time for all samples. ${ }^{13} \mathrm{C}$ NMR spectra with direct excitation were recorded with short interscan delays of $1 \mathrm{~s}$ to probe mobile components in the hydrogel sample. For heteronuclear decoupling during acquisition, SPINAL64 was employed with a $100 \mathrm{kHz}$ nutation 
frequency $\left({ }^{1} \mathrm{H}\right)$. The chemical shifts were referenced using adamantane (left signal at $38.48 \mathrm{ppm}$ ) by subsequent adjustment of the magnetic field.

\section{Raman spectroscopy}

The Raman spectra were recorded on an alpha $300 \mathrm{R}^{+}$confocal Raman microscope from WITec GmbH (Ulm, Germany) equipped with a 50x objective (NA 0.8, Epiplan Neofluar, Zeiss, Germany) and a 532 $\mathrm{nm}$ laser (39.4 mW). A $20 \mathrm{wt} . \%$ sample was measured after equilibration at $5{ }^{\circ} \mathrm{C}$ and $40{ }^{\circ} \mathrm{C}$ using a temperature controllable Peltier stage (LTS 120, Linkam Scientific Instruments Ltd., Tadworth, UK). Spectra are shown as average spectrum of 3 spectra at different locations on the same sample, which were recorded with an integration time of $5 \mathrm{~s}$ and 10 accumulations. The resulting data were processed with cosmic ray removal and background subtraction. The bulk water signals at $3100-3700 \mathrm{~cm}^{-1}$ were fitted using a Gaussian deconvolution method as described elsewhere..$^{28,29}$

\section{Fluorescence spectroscopy}

The amphiphilic fluorescence probe 2-(4-dimethylamino)styryl)-1-methylpyridinium iodide (Daspmi) was purchased from Molecular Probes Inc., Life Technologies. The hydrophobic probe 4,4'-Difluoro4bora-3a,4a-diaza-s-indacene meso-substituted with para-dodecylphenyl (BPC12) was synthesized like described in previous studies. ${ }^{30}$ Gibco $^{\text {TM }}$ Dulbecco's phosphate-buffered saline (DPBS) pH 7.25 was purchased from Thermo Fisher Scientific (Massachusetts, USA). The steady-state fluorescence spectra of Daspmi $(5 \mu \mathrm{M})$ and BPC12 $(5 \mu \mathrm{M})$ in aqueous sol and gel samples at a polymer concentration of 20 wt.\% were recorded at different temperatures on a FLS-1000 spectrofluorometer (Edinburgh Instruments, UK) equipped with a thermocontrolled cuvette holder ( $\lambda_{\text {exc. }}$ (Daspmi): $460 \mathrm{~nm}$,

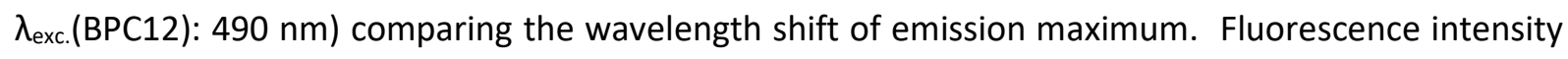
decay curves were obtained using a time-correlated single photon counting (TCSPC) system (PicoQUant $\mathrm{GmbH}$ ) equipped with a temperature controlling cuvette holder using quartz cuvettes like described earlier ( $\lambda_{\text {exc.: }} 483 \mathrm{~nm}$, cutoff filter $>490 \mathrm{~nm}$ ). ${ }^{31}$ Monitoring wavelengths were $580 \mathrm{~nm}$ for Daspmi and $520 \mathrm{~nm}$ for BPC12. The fluorescence decays were deconvoluted with the instrumental response to give the fluorescence lifetime with a resolution of approximately $100 \mathrm{ps}$. The obtained 
lifetimes were used for calculating micro viscosity values at different temperatures $(5,12,22$ and 37

$\left.{ }^{\circ} \mathrm{C}\right)$ using following equation:

$$
\log \tau_{f}=\log \frac{z}{k_{r}}+\alpha \log \eta
$$

with $\tau_{f}$ the fluorescence lifetime of Daspmi or BPC12 $2^{32}$ in the solution of a known viscosity $\eta, k_{r}-$ radiative rate constant, and $z$ and $\alpha$ are constants. The linear part of the $\log \tau_{f}$ as a function of $\log \eta$ plot was taken as a calibration function to calculate microviscosity values (Figure S3). The viscosities of different water/glycerol mixtures (80-100 wt.\% of glycerol) at different temperatures were determined using a LOVIS 2000M rolling ball microviscosimeter from Anton Paar (Graz, Austria) with a LOVIS 1.8 capillary and a steel ball of $1.5 \mathrm{~mm}$ diameter. Prior to viscosity measurements the density of the sample at the specific temperature was recorded using a DMA 4100M density meter from Anton Paar (Graz, Austria). For the corresponding lifetime measurements a concentration of $5 \mu \mathrm{M}$ of Daspmi was used.

Micro calorimetry $(\mu C A L)$

Micro calorimetry measurements were conducted with a Malvern MicroCal PEAQ-DSC microcalorimeter. The heat of the sample was measured relative to pure water and the enthalpy values were normalised to the molar concentration of repeating unit. The samples were stored after complete dissolution in a refrigerator at $4{ }^{\circ} \mathrm{C}$ for about $48 \mathrm{~h}$, degassed at $5{ }^{\circ} \mathrm{C}$, transferred to the instrument precooled at $2{ }^{\circ} \mathrm{C}$, and kept at $2{ }^{\circ} \mathrm{C}$ for 120 min prior to heating. Each sample was heated with the rate of $1{ }^{\circ} \mathrm{C} / \mathrm{min}$ from $2{ }^{\circ} \mathrm{C}$ to $100{ }^{\circ} \mathrm{C}$. The cooling back to $2^{\circ} \mathrm{C}$ was done with the same rate.

\section{Molecular modeling}

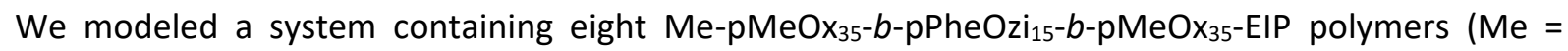
methyl group, EIP = ethyl isonipecotate), in which the hydrophobic pPheOzi blocks faced each other to form a single inner strand along the $\mathrm{Z}$ axis and the hydrophilic $\mathrm{pMeOx}$ blocks were bent outwards. Four individual molecules made up the strand and were subsequently duplicated and moved next to the original polymers along the $\mathrm{Z}$ axis, ultimately resulting in two layers of polymers in our simulation box. The stretched out, hydrophilic pMeOx blocks were subjected to an energy minimization and a short, 
50 ps long simulation with the Noisé-Poincaré-Andersen method ${ }^{33,34}$ (applying the Amber14:EHT force field ${ }^{35,36}$ with the R-field implicit solvation model ${ }^{37}$ ) to yield a more compact starting conformation, while keeping the inner strand in a straight orientation. Figure S6A depicts the prepared structure. All modeling was performed with MOE (Molecular Operating Environment 2019.01). ${ }^{38}$ The setup was inspired by previous modeling studies regarding worm-like micelles of small molecules, in which the generation of a continuous micelle was also achieved via initial placement of hydrophobic parts in the inner and hydrophilic moieties in the outer regions of the threadlike structure, which was aligned along one axis of the simulation box. ${ }^{39-41}$ RESP partial charges ${ }^{42}$ of single monomers used as building blocks were derived from calculations with Gaussian 09 Rev. C. $01^{43}$ (Hartree-Fock level of theory, 6-31G* basis set); parameters based on the Amber $14 \mathrm{ffSB}^{35}$ and GAFF2 ${ }^{44}$ force fields were assigned via antechamber and parmchk2 of AmberTools18. ${ }^{45}$ During charge derivation monomer structures were capped with residues of the same type (except for the terminal groups, Me and EIP, which were capped with a MeOx monomer). The calculated parameters were used to generate a polymer with an initial straight conformation using tleap. ${ }^{45}$

Subsequent solvation of the starting structure with TIP3P water ${ }^{46}$ in a simulation box with a minimum border-to-polymer distance of $20 \AA$ in the $\mathrm{X}$ and $\mathrm{Y}$ directions resulted in a system size of approximately $16 \times 18 \times 10 \mathrm{~nm}^{3}$ with 76730 solvent molecules. Water molecules found inside the inner hydrophobic strand after this initial placement were removed if the distance to the pPheOzi blocks was less than 10 Å. Periodic boundary conditions with minimum image convention were applied during the simulation, which allowed for an infinitely sized worm-like micelle along the $Z$ axis and ensured a sufficient distance between polymers of neighboring boxes along the $\mathrm{X}$ and $\mathrm{Y}$ dimensions. The simulation was performed using NAMD $2.13^{47}$ with $2 \mathrm{fs}$ time steps. An initial energy minimization of 10,000 steps was conducted before slowly heating the system from $100 \mathrm{~K}$ to $278 \mathrm{~K}$ over the course of $500 \mathrm{ps}$. Harmonic constraints were initially applied on all polymers and gradually reduced over an additional $1.6 \mathrm{~ns}$, allowing a rapid reordering of solvent molecules around the polymers. Langevin dynamics and the Nosé-Hoover Langevin piston method ( $1 \mathrm{~atm})$ were used for temperature and pressure control in an NPT ensemble. After another $2 \mathrm{~ns}$ of additional equilibration, the production run was performed for $600 \mathrm{~ns}$. Semi- 
isotropic coupling allowed for fluctuations along the $Z$ axis, independent from the $X$ and $Y$ axes. The particle mesh Ewald method ${ }^{48}$ with a cutoff of $1.2 \mathrm{~nm}$ was applied and coordinates were saved every 10 ps. Subsequent analyses were performed using cpptraj $^{49}$ and images were generated with VMD 1.9.3 $3^{50}$ and PyMOL 2.4.1. Average densities for polymer groups around PheOzi monomers were retrieved as follows: All PheOzi residues were iteratively aligned onto the same monomer. Next, binned occupancy histograms of different moieties around the center of the aligned residue were calculated for the last $100 \mathrm{~ns}$ using the grid command in cpptraj. This was performed on a $1.6 \mathrm{x} 1.6 \mathrm{x}$ $1.6 \mathrm{~nm}^{3}$ grid with a $1 \AA$ A resolution. After this procedure, the obtained values around each PheOzi monomer were added up at each grid element and divided by the number of analyzed frames (10000) and monomers (104). Thus, densities represent the average amount of atoms of interest found at each grid element per frame around a single monomer. The first and last PheOzi monomer of each pPheOzi block (16 out of 120) were excluded from this calculation, as these are always situated near the neighboring MeOx residues. Additionally, we analyzed several distances between these PheOzi monomers and the other polymer residues, as well as the angle $\omega$ between the plane of nearby amide $(\mathrm{N}-(\mathrm{C}=\mathrm{O})-\mathrm{C})$ groups and the phenyl ring plane for every $10 \mathrm{ps}$ of the last $100 \mathrm{~ns}$. 


\section{Results and Discussion}

As a first step to verify and better understand the self-assembly of $\mathrm{pMeO}_{35}-b$-pPheOzi ${ }_{15}-b-\mathrm{pMeO} x_{35}$, we used dynamic light scattering in aqueous solution $(0.1 \mathrm{~g} / \mathrm{L})$ at different temperatures $\left(15^{\circ} \mathrm{C}\right.$ and 40 $\left.{ }^{\circ} \mathrm{C}\right)$. At low temperature $\left(15^{\circ} \mathrm{C}\right.$, blue) the decay of the autocorrelation function (ACF) is shifted towards higher lag time indicating bigger particles with a lower translational diffusion coefficient compared to higher temperature $\left(40^{\circ} \mathrm{C}\right.$ ) (Figure $2 \mathrm{~A}$ ). For particles smaller than $\lambda / 20$ (here $632.8 \mathrm{~nm} / 20=32 \mathrm{~nm}$ ), the ACF is independent of the scattering angle. Therefore, similar values for the diffusion coefficient are obtained at $40^{\circ} \mathrm{C}$ for scattering angles between $20^{\circ}$ and $147^{\circ}$ (Figure $\mathrm{S} 1$, red). In contrast, at $15^{\circ} \mathrm{C}$ a clear angle dependency was observed (Figure S1, blue) indicating a larger diffusion coefficient at the gel temperature. By extrapolation to $0^{\circ}$ and applying the Stokes-Einstein relation, a hydrodynamic radius $\left(R_{h}\right)$ of $11.5 \pm 0.3 \mathrm{~nm}$ can be determined at $40{ }^{\circ} \mathrm{C}$. This corroborates the micellar sizes previously observed by cryoTEM for the same system. ${ }^{22}$ At $15{ }^{\circ} \mathrm{C}$, an approximately tenfold higher $\mathrm{R}_{\mathrm{h}}$ of $105 \pm 4$ $\mathrm{nm}$ is obtained, documenting a significant change in polymer self-assembly. It should be noted that these measurements were conducted at concentrations significantly lower than the critical gelation concentration $\left(c_{c r i t, g e l}=50 \mathrm{~g} / \mathrm{L}\right.$ ), at which cryoTEM images and $R_{\mathrm{h}}$ from DLS are not accessible. Therefore, additional temperature dependent SAXS and WAXS measurements above $c_{\text {crit,gel }}$ were performed (Figure 2B,C).

A)

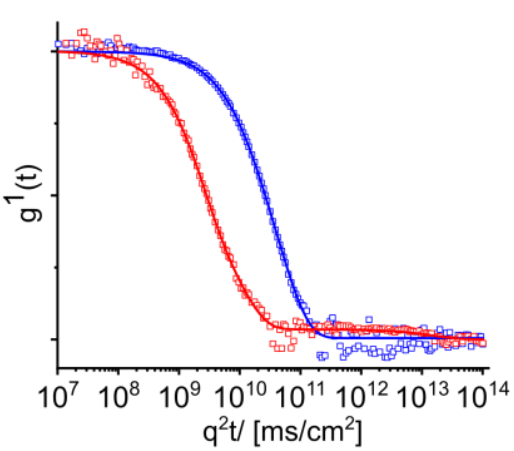

B)

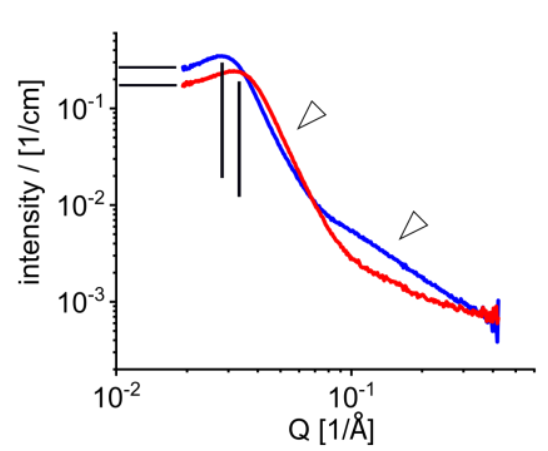

C)

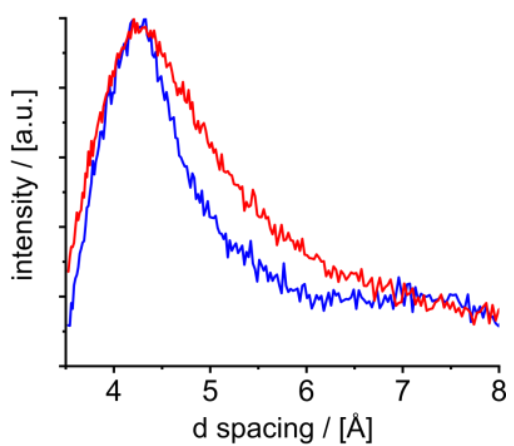

Figure 2| DLS, SAXS and WAXS analysis of aqueous pMeOx-b-pPheOzi-b-pMeOx solutions. A) Autocorrelation function $\mathrm{g}^{1}(\mathrm{t})$ as a function of $\mathrm{q}^{2} \mathrm{t}$ at $89^{\circ}$ scattering angle (squares: data points, lines: fit functions) at $15^{\circ} \mathrm{C}$ (blue) and $40^{\circ} \mathrm{C}$ (red) at $0.1 \mathrm{~g} / \mathrm{L}$. B) SAXS scattering profiles at $5^{\circ} \mathrm{C}$ (blue, hydrogel) and $40{ }^{\circ} \mathrm{C}$ (red, liquid) at $100 \mathrm{~g} / \mathrm{L}$. C) WAXS analysis at different temperatures (blue: $5^{\circ} \mathrm{C}$, red: $40^{\circ} \mathrm{C}$ ) in the $\mathrm{d}$ spacing region of $3.5-8 \AA$ at $100 \mathrm{~g} / \mathrm{L}$. 
The intensity I as a function of $Q$ from the SAXS measurements was plotted for different temperatures. In the hydrogel state $\left(5^{\circ} \mathrm{C}\right.$, blue), a pronounced structure peak (Figure $2 \mathrm{~B}$ ) is followed by two clearly defined regions with different slopes (arrowheads). These regions at intermediate and high Q-values can be assigned to different self-assembled species. Using a power-law expression the different slopes can be determined, indicating the presence of spherical micelles and worm-like micelles in the gel state. ${ }^{51}$ Above $T_{\text {gel, }}$ the second species at high Q-values (worm-like micelles) disappears, confirming that the order-order transition found at low concentration also occurs at higher concentration. In addition, the structure peak is shifted towards higher Q-values indicating a lower particle/particle distance in the sol state due to the formation of small spherical micelles (vertical lines). This is a logical consequence of the disintegration of relatively few worm-micelles into much more numerous spherical micelles. The extrapolation of the absolute intensity I to $Q_{0}$ (horizontal lines) was used as a measure for relative mean particle size. In the gel state $\left(5^{\circ} \mathrm{C}\right)$, a higher $\mathrm{I}\left(\mathrm{Q}_{0}\right)$ value compared to the liquid state was observed, indicating once more larger particles in gel state (worm-like micelles) in comparison to the sol state (spherical micelles). The qualitative analysis of the SAXS scattering profiles is summarized in more detail in the supporting information (Table S1). Additionally, temperature-dependent wide angle X-ray scattering (Figure $2 \mathrm{C}$ ) can provide insights into intra- und interpolymer interactions as previously described for biopolymers ${ }^{52}$ and thermogelling peptides ${ }^{53}$. In the gel state $\left(5^{\circ} \mathrm{C}\right.$, blue), a rather defined peak centered around $4.2 \AA$ was observed, which could be interpreted to hint towards $\pi-\pi$ interactions of the phenyl moieties. ${ }^{54}$ With increasing temperature, the peak position was maintained, but a noticeably broadening between 4.2 and $7 \AA$ indicates reduced order and increased degrees of freedom.

In the next step, micro-calorimetric measurements were performed to gain a better insight into thermodynamic aspects of this order-order transitions. When an equilibrated hydrogel sample (stored $48 \mathrm{~h}$ at $5{ }^{\circ} \mathrm{C}$ ) was examined, a single endothermic peak is observed at $31.2^{\circ} \mathrm{C}$ with an enthalpy $\mathrm{dH}$ of $0.47 \mathrm{~kJ} / \mathrm{mol}$ per aromatic repeat unit of pPheOzi (Figure 3), coinciding with the gel-sol transition temperature. ${ }^{22}$ Notably, during the cooling cycle no features were observed (not shown). 


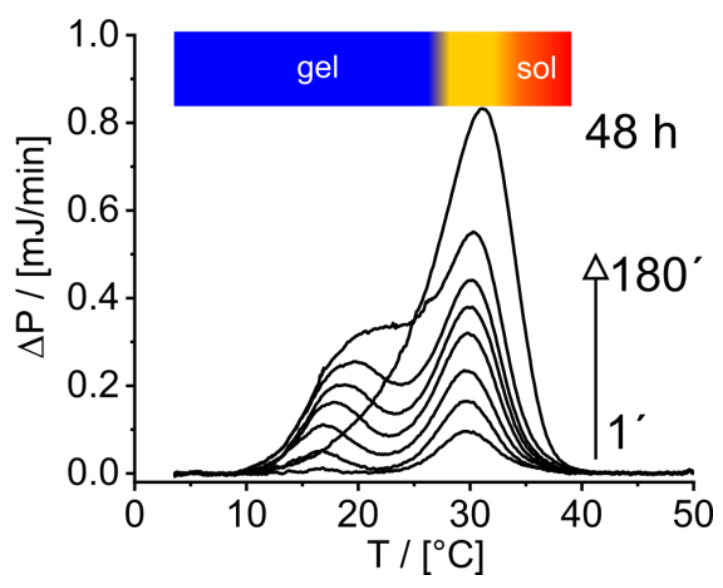

Figure 3| Micro-calorimetry measurements of a $10 \mathrm{~g} / \mathrm{L}$ sample. The sample was equilibrated for $48 \mathrm{~h}$ at $5{ }^{\circ} \mathrm{C}$ prior to measurement. After an initial heating and cooling the sample was kept at $2{ }^{\circ} \mathrm{C}$ for different times $(1-10$ 20-40-60-90-180 minutes) to visualize the time dependent hydrogel formation.

When immediately reheated, a much weaker transition was observed. When the sample was kept for some time at $2{ }^{\circ} \mathrm{C}$, a second peak at lower temperature emerged (initially at $17^{\circ} \mathrm{C}$ ) and the intensity of both peaks increased with increasing wait time. Interestingly, the maximum of the second peak shifts to higher temperatures with increasing regelation time at $2{ }^{\circ} \mathrm{C}$ after each heating and cooling cycle. In contrast, the peak maximum of the first peak remains constant at $31{ }^{\circ} \mathrm{C}$. This observation coincides with the delayed gelation upon cooling in dependence of the concentration and temperature. ${ }^{22} \mathrm{We}$ hypothesize that the first peak can be attributed to a transition in single spherical micelles or short worms. As the short worms slowly coalesce into larger worms, the transition temperature increases. The second transition is then attributed to worms, which grew above a certain threshold, above which further growth does not affect the transition temperature anymore. Thus, we have established an order-order transition coinciding with a change in macroscopic properties. From a self-assembly point of view, a transition from spherical to worm-like assembly must correlate with the change in the volume fraction of the hydrophilic to the hydrophobic compartment. Specifically, the hydrophilic pMeOx block would have to decrease in volume or the hydrophobic pPheOzi block would have to increase in volume upon cooling for a sphere-to-worm transition to occur. However, neither blocks are known to be thermoresponsive in this case, so it is not apparent a priori, what causes this order-order transition on a (macro)molecular level. 
An indirect method to determine (macro)molecular mobility and polymer self-assembly is the use of viscosity sensitive fluorescent probes, namely molecular rotors such as 4,4'-difluoro-4-bora-3a,4adiaza-s-indacene meso-substituted with para-dodecylphenyl, BODIPY-C12 (BPC12) and 2-(4dimethylamino)styryl)-1-methylpyridinium iodide, Daspmi (Figure S2). ${ }^{31}$ The fluorescence lifetime of a molecular rotor is affected by a rotation ability of its structural segments with respect to each other, which is strongly dependent on the immediate molecular environment. However, it has to be kept in mind that it is not always clear what this immediate molecular environment is in the presence of selfassembled species. Here, microviscosities in the sol and gel states were determined by fluorescence lifetime data after appropriate calibration (Figure $\mathrm{S} 3)^{32}$ at four temperatures $\left(5,12,22\right.$ and $\left.37^{\circ} \mathrm{C}\right)$. We intended to probe the temperature-dependent microviscosity of both, hydrophobic and hydrophilic domains of the self-assemblies by using two different rotors, one being more hydrophobic (BPC12) and the other being more hydrophilic/amphiphilic (Daspmi). Interestingly, in contrast to bulk viscosity (macroviscosity) of the polymer that is obviously higher in gel state compared to sol state, the microviscosity decreased upon gelation. The decrease for the hydrophilic probe was much more pronounced, suggesting more substantial changes in its microenvironment and therefore of the hydrophilic compartment upon gelation (Figure 4A). In the gel state, the microviscosity was similar for both probes. In contrast, in the sol state the more hydrophilic Daspmi experienced a microviscosity that was almost three times higher compared to BPC12.
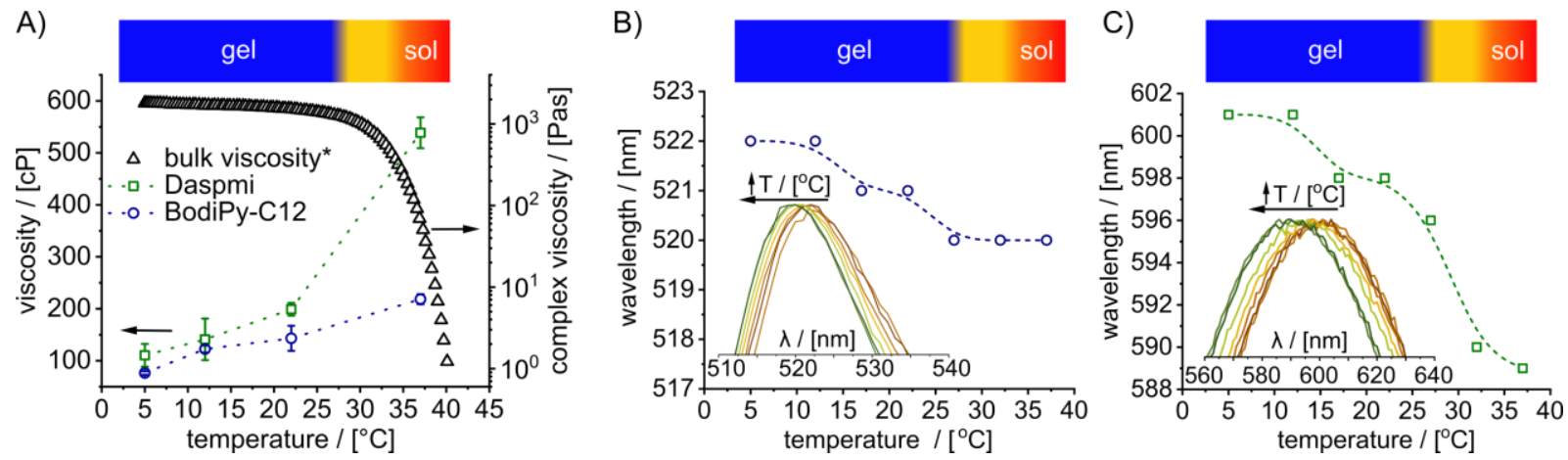

Figure 4| Microviscosity and polarity change investigated using molecular rotors and fluorescence spectroscopy. A) Microviscosity values for Daspmi and BPC12 molecular rotors in a 20 wt.\% aqueous polymer sample in fluorescence lifetime experiments as a function of temperature. Bulk viscosity of a 20 wt.\% hydrogel was added for comparison using the data obtained in previous studies via oscillatory rheology experiments. ${ }^{22}$ Wavelength shift of BPC12 (B) and Daspmi (C) fluorescence emission in steady-state experiments as a function of temperature. 
The result can be interpreted so that in the sol state, where spherical micelles are present, the used rotors are located in different self-assembly domains, presumably Daspmi stays within the hydrophilic corona and BPC12 in the hydrophobic core of the micelles. We would like to remind the reader that this must concur with a decrease of the volume of the hydrophilic corona or an increase of the volume of the hydrophobic compartment. Thus, upon order-order transition from spheres to worms, Daspmi appears to be expelled from the corona. Steady-state fluorescence spectroscopy complements the picture. Fluorescence intensity and $\lambda_{\max }$ shift can provide information on polarity changes of the probe's microenvironment. BPC12 and its derivatives are known to exhibit a lower degree of responsiveness in this context compared to Daspmi. ${ }^{55}$ For both probes, a two-step bathochromic shift was observed at low temperature, indicating an increase in polarity upon gelation (Figure 4B,C). This increase supports the suggestion that the probes can be partially expelled from polymeric selfassemblies and become more exposed to polar aqueous solvent. Interestingly, the first polarity change is at a temperature between $12^{\circ} \mathrm{C}$ and $17{ }^{\circ} \mathrm{C}$, which coincides with the first endothermic peak observed in micro-calorimetry (Figure 3). Again, the change was much more pronounced for Daspmi. Ultimately, the time-resolved and steady-state fluorescence measurements clearly show that the microenvironment of both molecular rotors is more polar and less viscous in the gel state, suggesting that the gelation causes a probe migration out of the condensed polymeric assembly closer to the polymer-water interface.

Raman spectroscopy can provide functional group-selective information on covalent and non-covalent polymer interactions. Importantly, it is well suited for the characterization of highly concentrated aqueous systems and can allow drawing conclusions about existing conformations. ${ }^{47}$ Accordingly, we investigated a $20 \mathrm{wt} . \%$ polymer sample at $5{ }^{\circ} \mathrm{C}$ (gel) and $40{ }^{\circ} \mathrm{C}$ (sol) and compared this to the dried polymer powder (Figure 5). 


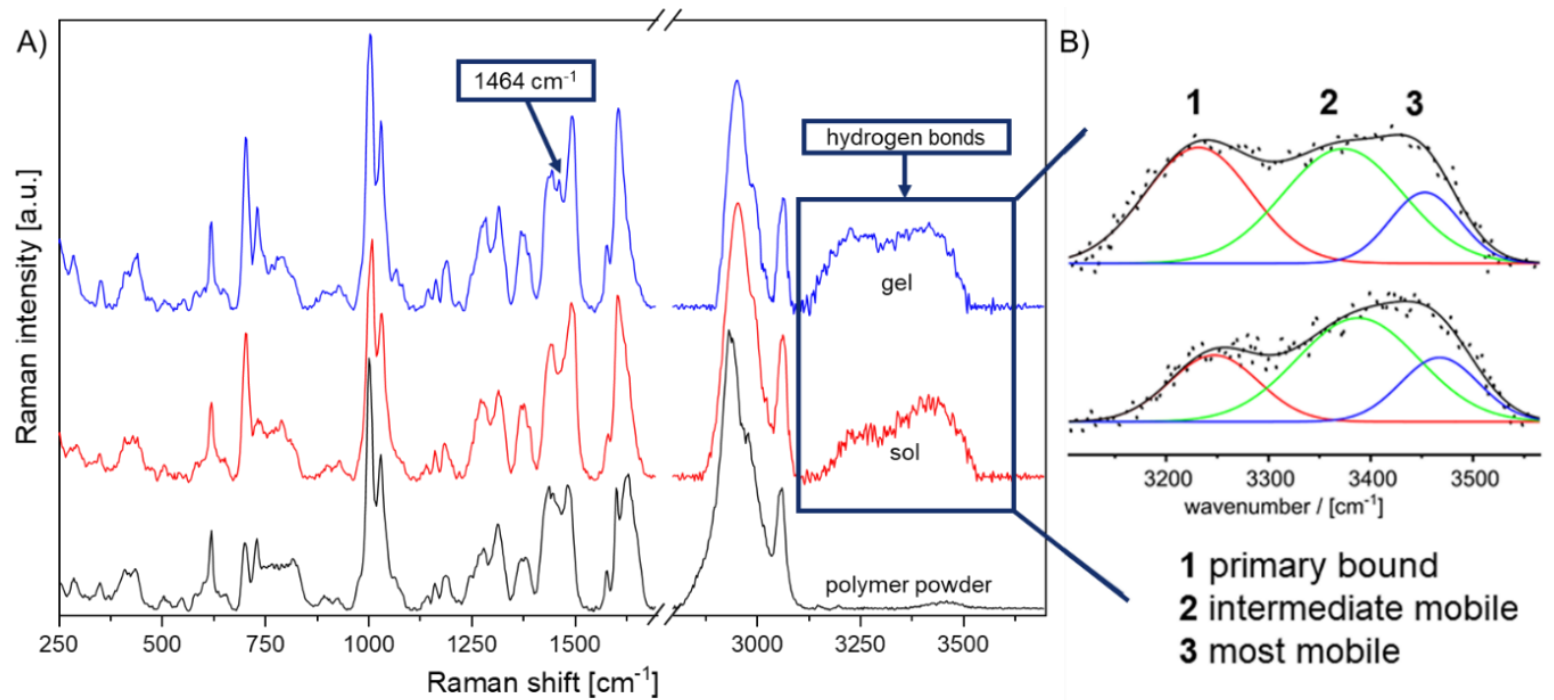

Figure 5| Temperature dependent Raman experiments of a $20 \mathrm{wt} . \%$ sample in sol $\left(40^{\circ} \mathrm{C}\right)$ and gel $\left(5^{\circ} \mathrm{C}\right)$ state. A)) Normalized Raman spectra $\left(250-3700 \mathrm{~cm}^{-1}\right)$ of dried polymer powder (black), polymer sol $\left(40{ }^{\circ} \mathrm{C}\right.$, red) and polymer gel $\left(5^{\circ} \mathrm{C}\right.$, blue). At $1464 \mathrm{~cm}^{-1}$ a peak appears in the gel state, with additional changes in the signal pattern in the region of hydrogen bonds involving water molecules $\left(3100-3600 \mathrm{~cm}^{-1}\right)$. B) Deconvolution of the OH-region of the spectrum (dashed line) using the Gaussian deconvolution method showing the individual Gaussian peaks (colored lines) and their sum (black line). The different possible hydrogen bonds are describing different types of water, which can be assigned to primary bound, intermediate mobile, and most mobile water molecules.

Overall, the Raman spectra for the dried polymer powder, polymer hydrogel $\left(20 \mathrm{wt} . \%, 5{ }^{\circ} \mathrm{C}\right)$ and polymer sol (20 wt.\%, $40^{\circ} \mathrm{C}$ ) show similar features (Figure 5B-D), yet minor differences further corroborate changes in the polymer-polymer interactions between sol and gel state as observed by WAXS. At $1464 \mathrm{~cm}^{-1}$, a small but clearly distinguishable peak is exclusively observed in the gel, but unambiguous assignment of this signal is challenging. In this region both aromatic ring vibrations as well as $\mathrm{CH}_{3}$ and $\mathrm{CH}_{2}$ deformation vibrations appear, and these are ubiquitous in the polymer structure. A more profound difference between sol and gel state is observed in the $\mathrm{OH}$ region of $3100 \mathrm{~cm}^{-1}$ to $3600 \mathrm{~cm}^{-1}$, which in this system can only originate from water molecules, since no significant signal is detectable in the lyophilized polymer powder in this region. The different types of bonding modes in water molecules can be categorized using Gaussian deconvolution. In general, due to the wider energy gap between vibration modes, Raman bands of strong chemical bonds have lower Raman shifts. This can be used to divide the $\mathrm{OH}$ region into areas with different binding strength as previously described by various groups. ${ }^{28,56,57}$ Raman shifts between 3200 and $3300 \mathrm{~cm}^{-1}$ indicate water molecules with more hydrogen bonds and are therefore considered primary bound water. Increasing shifts are interpreted as intermediately (3300-3400 $\left.\mathrm{cm}^{-1}\right)$ with fewer hydrogen bonds and strongly mobile $\left(3450-3550 \mathrm{~cm}^{-1}\right)$ 
water molecules. In the present system, $\mathrm{H}$-bond donors and acceptors can originate from other water molecules, while in the polymer only $\mathrm{H}$-bond acceptors are present. Our data suggest that water is more strongly bound/less mobile in the hydrogel compared to the sol (Figure 5D) as indicated by the increased contribution of the peak at $3250 \mathrm{~cm}^{-1}$ (red line, 1). While we see specific differences in the Raman spectra between sol and gel state, a specific assignment of polymer-polymer interactions responsible for the order-order transition remains elusive, and additional analytical tools are needed.

Due to its sensitivity to short-range order phenomena and more straightforward assignability of signals to specific moieties, NMR spectroscopy is a versatile tool to further study the underlying phase transitions. In a first step, all signals in the ${ }^{1} \mathrm{H}$ NMR experiments were assigned to the functional groups of the polymer (Figure $6 \mathrm{~A}, \mathrm{~B}$ ). Notably, the aromatic protons in the sidechain of the hydrophobic polymer block show a broad signal at 6.6-7.6 ppm (signal 5), which differs significantly in appearance between sol and gel state (Figure 6B). Four signal areas around 6.9, 7.2, 7.4 and 7.5 ppm (signals 5.15.4) can be distinguished. In the hydrogel state $\left(2{ }^{\circ} \mathrm{C}-31^{\circ} \mathrm{C}\right)$, an overall low signal intensity with a relatively defined peak at $7.5 \mathrm{ppm}$ and a broad shoulder around $7.4 \mathrm{ppm}$ is observed. Increasing the temperature above the sol/gel transition $\left(34{ }^{\circ} \mathrm{C}-40{ }^{\circ} \mathrm{C}\right.$ ) results in significantly increased signal intensities and reduced line widths in line with the expected mobility increase for the polymer chains. The signals in the aliphatic region (backbone and $\mathrm{CH}_{3}$ group of $\mathrm{MeOx}$, signals 1-4) also become much more defined. The fraction $p$ (equation (3)) allows a more quantitative assessment of the relative decrease of the respective peak areas, which allows drawing conclusions about the mobility of the associated polymer segments (Figure 6C). 

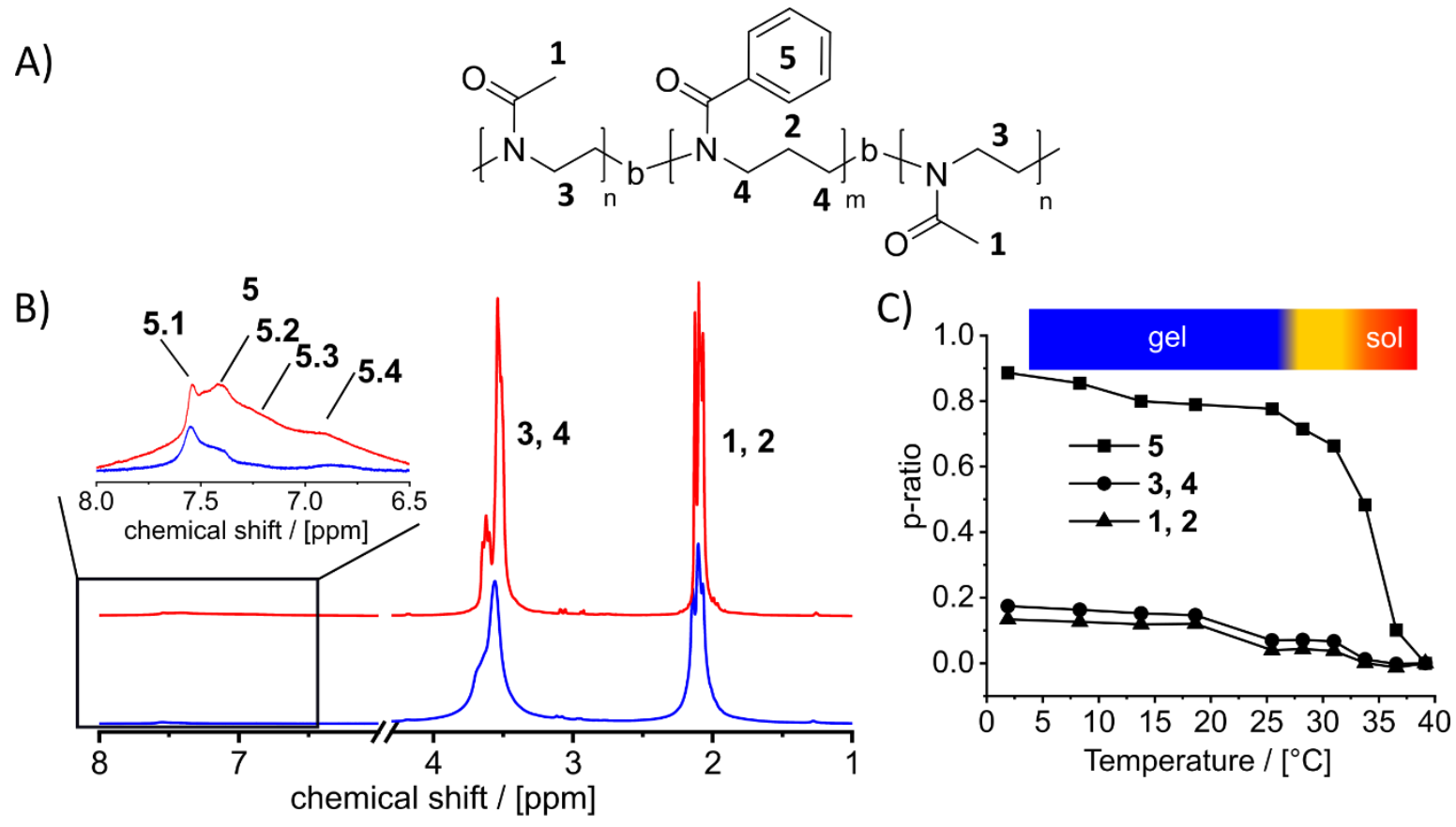

Figure $\left.6\right|^{1} \mathrm{H}$ NMR experiments of a $20 \mathrm{wt} . \%$ sample in $\mathrm{D}_{2} \mathrm{O}$ recorded at different temperatures. A) Structure of the amphiphilic ABA type triblock copolymer pMeOx-b-pPheOzi- $b$-pMeOx including numbering scheme. B) ${ }^{1} \mathrm{H}$ NMR spectra at $2{ }^{\circ} \mathrm{C}$ (blue) and $40^{\circ} \mathrm{C}$ (red) of a pMeOx-b-pPheOzi-b-pMeOx sample alongside signal assignment. C) Calculated $p$-ratio for the intensities of different polymer protons as a function of temperature.

The aromatic $\mathrm{CH}$-protons yield the highest $p$-values in the gel-state. Upon liquefaction, the $p$-value decreases drastically indicating a more flexible and mobile hydrophobic micellar core for the spherical micelles. Overall, the hydrophilic block and the backbone are less affected at worm formation corroborating results by Weberskirch et al. and Černoch et al. describing thermoresponsive POx based homo- and copolymers. ${ }^{27,58} \mathrm{~A}$ first decrease in $p$-value occurred at $15-20{ }^{\circ} \mathrm{C}$, followed by a plateau region $\left(20-30^{\circ} \mathrm{C}\right)$, before the $p$-value drops to zero upon liquefaction. This observation agrees with the results obtained by micro-calorimetry and steady state fluorescence. Mobility information for the different moieties can also be obtained through the comparison of ${ }^{1} \mathrm{H}$ longitudinal relaxation times $\mathrm{T} 1$, which were determined for the sol and gel state, respectively (Figure S4, Table S2). The fact that all polymer segments yield the same trend of decreasing T1-values upon increasing temperature supports the assumption that all parts of the polymer act in concert in the aggregation process. The same trend can be drawn for the water signal, since a high T1 value for small molecules in the sol state indicates more mobile water molecules compared to the gel state. This supports the Raman and steady state fluorescence results discussed earlier. Before the hydrophobic aromatic core of the worm like micelle 
becomes much more mobile, the hydrophilic block and the polymer backbone become more flexible. However, it is only when the aromatic block undergoes a drastic increase in mobility (in conjunction with a minor increase of the hydrophilic block and polymer backbone) that the order-order transition from worm to spherical micelle is observed. Regarding the necessary change in volume ratio of hydrophilic and hydrophobic blocks, the much more pronounced reduction in mobility of the hydrophobic block would suggest a compaction rather than a volume increase. This leaves only the possibility of a significant volume decrease of the hydrophilic block. Even though the reduction of mobility is much less pronounced, the reduced mobility does hint to some compaction of the hydrophilic domain.

To obtain more insights into the spatial proximity between different moieties in the polymer, $2 \mathrm{D}^{1} \mathrm{H}-{ }^{1} \mathrm{H}$ NOESY NMR experiments were performed in the gel $\left(5^{\circ} \mathrm{C}\right)$ and sol $\left(40^{\circ} \mathrm{C}\right)$ state (Figure 7$)$. Notably, significantly stronger NOE signals are visible in the hydrogel state (Figure 7A) compared to the sol (Figure 7B), which indicates on average a closer proximity of the individual polymer moieties and agrees with the results from WAXS (Figure 1C).

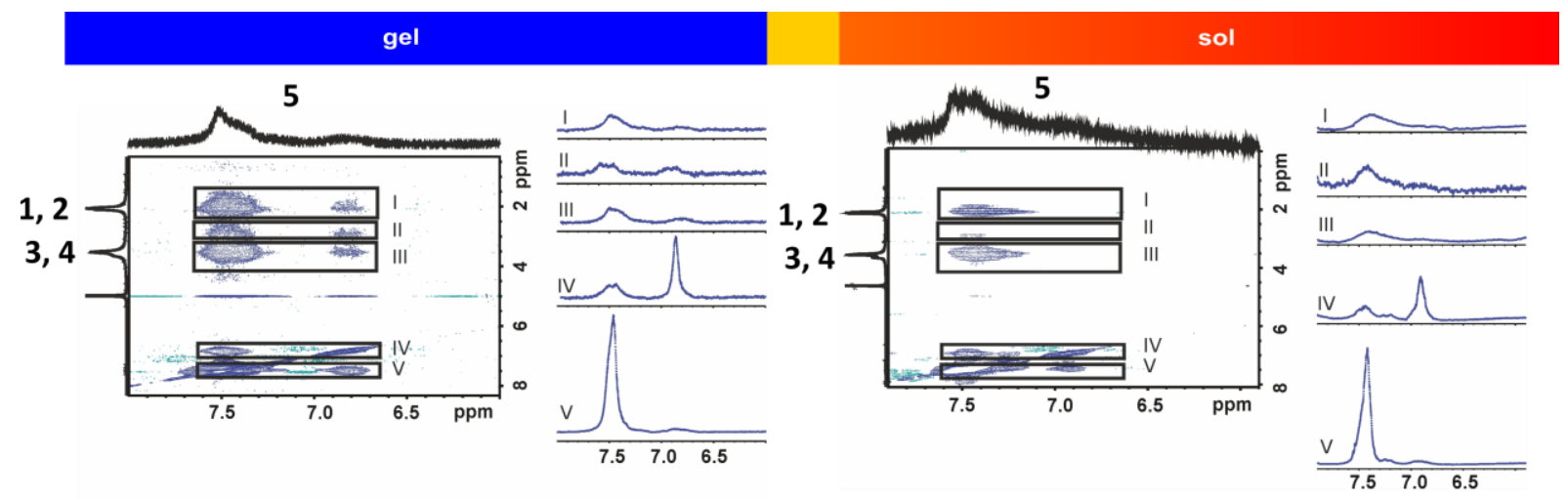

Figure $7{ }^{1} \mathrm{H}-{ }^{1} \mathrm{H}$ NOESY NMR experiments of a 20 wt.\% sample in $\mathrm{D}_{2} \mathrm{O}$ recorded with a mixing time of $40 \mu \mathrm{s} .5$ ${ }^{\circ} \mathrm{C}$ (blue, gel state, worm like micelle). $40{ }^{\circ} \mathrm{C}$ (red, sol state, spherical micelle). For signal areas of interest 1D slices were extracted and the assignment in figure 6 for the specific polymer signals was used.

In the sol state, a single intense NOE cross peak originating from the defined aromatic peak at $7.5 \mathrm{ppm}$ can be correlated to the backbone and $\mathrm{MeOx}$ sidechain protons. In the gel state an additional second cross peak of the aromatic region at $6.9 \mathrm{ppm}$ with the backbone and $\mathrm{MeOx}$ sidechain protons is evident despite the overall lower ${ }^{1} \mathrm{H}$ signal intensity in this area compared to the sol state. The observation of 
an additional NOE signal and, therefore, increased spatial proximity of aromatic and hydrophilic units is a first but crucial hint at a possible molecular mechanism to explain the unique assembly of this amphiphile into worm-like micelles at lower temperature. If the hydrophilic blocks interact with the hydrophobic block in any significant manner upon cooling, this could reduce the hydrophilic/hydrophobic volume ratio and lead to the order-order transition.

Considering their nature as viscoelastic solids, hydrogels can also be characterized using solid-state NMR experiments. ${ }^{59}$ For shear thinning hydrogels such as the present system, one has to consider the possibility that magic angle spinning (MAS) can exert sufficient force to alter the samples properties, e.g. causing liquefaction. This is obviously dependent on the yield point of the hydrogel and the extend of shear thinning the material undergoes. Accordingly, preliminary ${ }^{1} \mathrm{H}$ NMR experiments at different MAS frequencies (3-5.3 kHz) were recorded. No significant differences were observed (Figure S5). Two different ${ }^{13} \mathrm{C}$ NMR spectra were recorded either through direct excitation (DE) with short interscan delay (1s) showing predominantly mobile carbon environments (Figure $8 \mathrm{~A}$, grey spectrum), while ${ }^{1} \mathrm{H}$ ${ }^{13} \mathrm{C}$ cross polarization (CP) MAS experiments with a contact time of $2 \mathrm{~ms}$ showcase more rigid molecular entities due to their dependence on dipolar interactions (Figure 8A, black spectrum). In the spectrum obtained through $\mathrm{DE}$, only signals that can be attributed to $\mathrm{MeOx}$ units were visible and no aromatic signals were observed, corroborating once more the reduced mobility of these building blocks. In the ${ }^{13} \mathrm{C}$ CP MAS NMR spectrum significantly more and broader peaks as well as spinning sideband (indicated by asterisks) were observed with phenyl moieties now clearly observable. A MAS rate of 5 $\mathrm{kHz}$ or higher is required to avoid substantial overlap between the carbonyl signal of the amide group and the phenyl spinning sidebands. The broader signal (compared to DE spectra) of the amide CO group is slightly shifted to smaller ppm values (orange arrow) and a band of additional signals is visible at higher ppm originating from the phenyl carbonyl groups. Most interesting, however, are two additional signals that are observed in the aliphatic region of the spectrum (red arrows). The signal at $38 \mathrm{ppm}$ is in proximity to the other backbone signals indicating a similar chemical environment, while another 
new and broad signal appears at $24 \mathrm{ppm}$ adjacent the signal of the methyl sidechain of the hydrophilic polymer block.

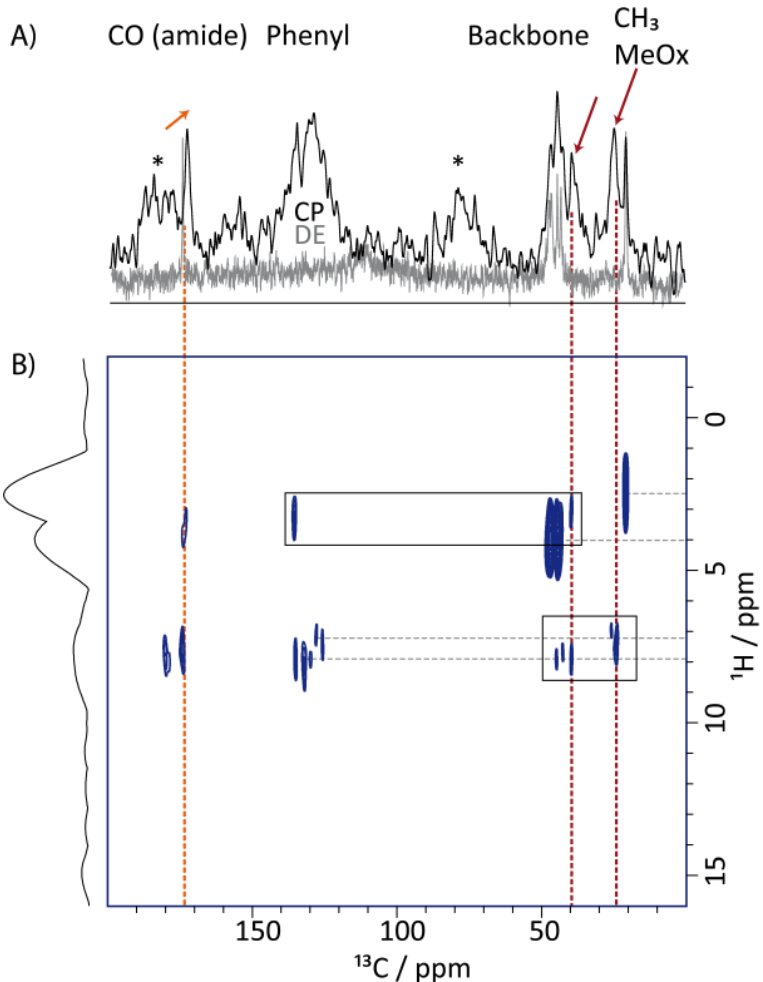

Figure 8| Solid state NMR experiments of a 20 wt.\% hydrogel sample. A) Overlay of the ${ }^{13} \mathrm{C}$ NMR spectra recorded at $9.4 \mathrm{~T}$ and $5.3 \mathrm{kHz}$ MAS using DE and short interscan delay of $1 \mathrm{~s}$ (grey) or CP MAS with 2 ms contact time (black). Spinning sidebands are indicated by asterisks. B) ${ }^{1} \mathrm{H}-{ }^{13} \mathrm{C}$ HETCOR MAS spectrum recorded at $9.4 \mathrm{~T}$ and a MAS rate of $5 \mathrm{kHz}$ using a contact time of $2 \mathrm{~ms} .122 \mathrm{t}_{1}$ FID increments were acquired using a recycle delay of $2 \mathrm{~s}$, each with 240 co-added transients. The corresponding ${ }^{13} \mathrm{C}$ NMR spectra are shown at the bottom. Direct $\mathrm{CH}$ contacts are indicated by dotted grey lines.

To better understand the nature of these new signals and investigate proximities through space in more detail, a 2D ${ }^{1} \mathrm{H}-{ }^{13} \mathrm{C}$ HETCOR experiment with a contact time of $2 \mathrm{~ms}$ was recorded (Figure 8B). Due to the relatively long contact time, intra- and intermolecular proximities can be observed in the 2D spectrum as cross peaks. The ${ }^{1} \mathrm{H}$ chemical shifts of the $\mathrm{CH}_{3}$ groups of MeOx, the backbone, and the two major phenyl environments are indicated by grey dotted, horizontal lines. The three signals highlighted in Figure 8A are indicated by red dotted, vertical lines. For the carbonyl signal, cross peaks both in the aromatic and aliphatic backbone region are observed, but due to the polymer structure we cannot know whether the proximity is intramolecular or intermolecular. Furthermore, cross peaks between some of the backbone $\mathrm{CH}_{2}$ signals and the phenyl signal at higher ppm are also visible (black boxes). Considering the larger intramolecular distance between backbone and the phenyl ring, this 
interaction is most likely through space. For the remaining carbon signals in the aliphatic region, it is clear that interactions must occur through space. The more rigid $\mathrm{CH}_{3}$ group of $\mathrm{MeOx}$ at $24 \mathrm{ppm}$ only visible in the CP-MAS experiment is in a different polymer block than the phenyl moiety. Therefore, the cross peak at $24 / 7.2 \mathrm{ppm}$ can only be explained by ${ }^{1} \mathrm{H}-{ }^{13} \mathrm{C}$ proximity through space $(<4 \AA$ ) between the hydrophilic and the hydrophobic block. In contrast, the more mobile $\mathrm{CH}_{3}$ group of MeOx at slightly lower ppm values does not show any cross peaks in the 2D correlation and thus represents the hydrated hydrophilic corona. With this, we can finally formulate a mechanism for the order-order transition and inverse thermogelation in aqueous solutions of $\mathrm{pMeO}_{35}-\mathrm{pPheOzi}_{15}-\mathrm{pMeO}_{35}$. Our results show that this thermogelation is due to an unexpected interaction between repeat units in the hydrophilic pMeOx blocks and those in the aromatic hydrophobic pPheOzi block. Polymers that show UCST-type thermotransition typically exhibit $\mathrm{H}$-bonding between polymer chains which is impossible in the present case. So what is the origin of this interaction? A look into the literature yields two most probable candidates, the overlap of the lone electron pairs of the amide carbonyl and the aromatic LUMO: $\mathrm{n}_{\mathrm{Am}} \bullet \bullet \pi^{*}{ }_{\mathrm{Ar}}$ or interactions of the $\pi$-orbitals of the amide and phenyl ring: $\pi_{\mathrm{Am}} \bullet \bullet \bullet \pi_{\mathrm{Ar}}$. In general, non-covalent $n \rightarrow \pi^{*}$ interactions have been described to contribute to the thermostability of the proline-rich protein collagen. ${ }^{60}$ Proline is the only proteinogenic amino acid that forms tertiary amides akin to the amide groups in POx and POzi. In addition, and even more closely related to the present system, $\mathrm{n} \rightarrow \pi^{*}{ }_{\mathrm{Ar}}$ interactions have been described to contribute to the structure formation in peptoids ${ }^{61}$, which again contain tertiary amides. The analytically rather elusive $n \rightarrow \pi^{*}$ Ar interactions are typically verified by crystallographic data or computational modeling. While the former can be ruled out for our system, we performed an all-atom molecular dynamics (MD) simulation of a single wormlike micelle consisting of full length $\mathrm{pMeOx}_{35}-b$-pPheOzi ${ }_{15}-b$-pMeOx ${ }_{35}$ amphiphiles at $5{ }^{\circ} \mathrm{C}$ (Figure S6A). We modeled the pPheOzi blocks as a central inner strand, which is surrounded by the corresponding pMeOx blocks stretching out into the solvent (water). Throughout the $600 \mathrm{~ns}$ simulation a single wormlike strand of pPheOzi blocks is preserved. More interestingly, the peripheral pMeOx blocks approach the initially solvent-exposed hydrophobic repeating units (Figure 9A,B), clearly corroborating our model of a hydrophilic shell condensing onto the hydrophobic core. 


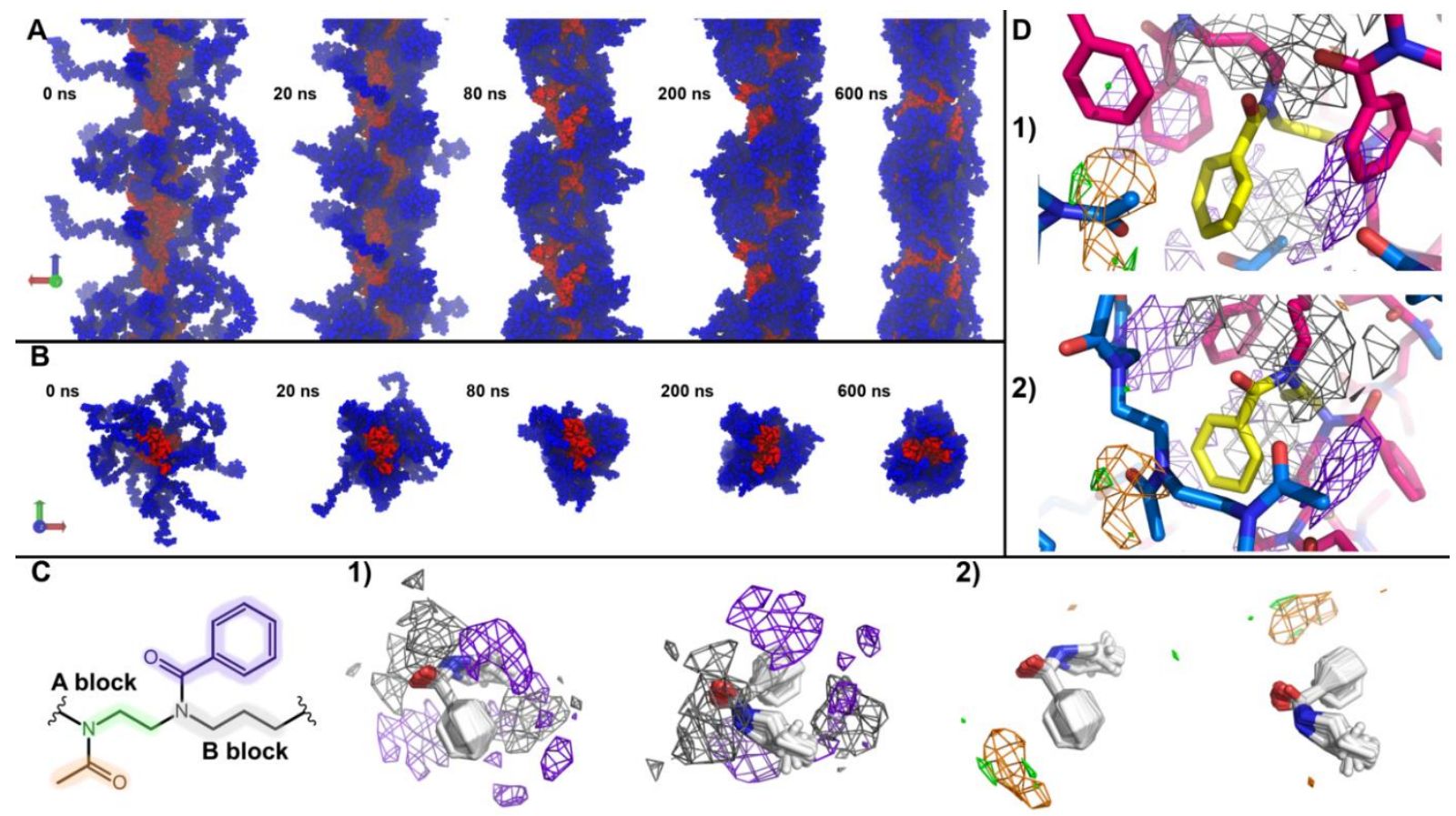

Figure 9| Results of molecular modeling of a worm-like micelle comprising pMeOx-b-pPheOzi- $b$-pMeOx amphiphiles. A) Simulation snapshots showing PheOzi monomers as red and MeOx monomers as blue VDW spheres. The simulation box and about half of each neighboring image along the $Z$ axis are illustrated, without solvent molecules depicted. B) Same illustration as seen in A) from an orthogonal perspective (along the $Z$ axis). C) Occupancy density analysis around aligned PheOzi residues (white sticks), showing hotspots for different polymer structures as meshes from two different perspectives. In C-1 the violet density represens PheOzi side chains and the grey density PheOzi backbone atoms (isovalues: 0.08). The two structures on the right in C-2 depict densities (isovalues: 0.03) for MeOx backbone atoms (green) and side chain atoms (orange) from two different perspectives. D) Illustrates two example snapshots D-1 and D- 2 in which residues at the surface of the micelle overlap with occupancy hot spots from $\mathrm{C}$ ). pMeOx residues are shown with blue carbon atoms, pPheOzi residues with magenta carbon atoms and the aligned monomer of interest is highlighted in yellow. Densities are shown analogously to $\mathrm{C}$ ).

Consequently, the radius of gyration of the self-assemblies decreases quickly and reaches a narrow fluctuation range after about $40 \mathrm{~ns}$ (Figure S6B, left). While the overall structure becomes more compact, not all pMeOx repeat units come into close contact with pPheOzi residues, which in turn are also not completely shielded from the solvent at the end of the simulation, corroborating the observations by solution NMR spectroscopy. In silico, about $53 \%$ of all pMeOx repeat units keep a minimum average distance of more than $5 \AA$ to the pPheOzi blocks over the last 100 ns (including hydrogen atoms for calculation). These would be attributed to the pMeOx repeat units, which were found more mobile in the ${ }^{13} \mathrm{C}$ DE MAS NMR spectra and which did not show cross peaks with the aromatic rings of pPheOzi in the ${ }^{1} \mathrm{H}-{ }^{13} \mathrm{C}$ HETCOR spectrum. We analyzed the solvent accessible surface area of all pPheOzi units and noticed that this value is mostly decreasing early on for the aromatic side 
chains, indicating that $\mathrm{pMeOx}$ monomers are preferably shielding these moieties against water molecules (Figure S6B, middle). The amount of water within $5 \AA$ around polymer residues also decreases quickly, especially for the pMeOx monomers (Figure S6B, right). An illustration of the average occupancy densities of polymer moieties around pPheOzi moieties supports this observation (Figure 9B). pMeOx and, more specifically, their side chains are predominantly located close to the aromatic ring of pPheOzi side chains in near vicinity to the pPheOzi carbonyl group. In contrast, aromatic rings of pPheOzi can be found below or above other pPheOzi amide groups and near the phenyl ring in the area which is turned away from the carbonyl group. The pPheOzi backbone atoms are mainly surrounded by other pPheOzi residues. Interestingly, a small fraction of the aromatic repeat units remains solvent-exposed: 17 out of 120 pPheOzi repeating units show an average minimum distance of over $3 \AA$ to any pMeOx in the last 100 ns (taking hydrogen atoms into account). These could be interpreted as sticky patches, which help to mechanically connect different worm-like micelles, adding to the remarkably high storage modulus of the gels. ${ }^{22}$ The evidence of such sticky contacts in worm-like micelles was recently discussed by Thurn and Hoffmann. ${ }^{62}$ On the other hand, this could also result from an insufficient number of polymers in our model, as the exact composition of the micelle was not available as input $a$ priori from experimental data. For the pPheOzi residues analyzed during density calculation, we measured distances to the nearest polymer moieties, as well as the angle $\omega$ between the planes of nearby $\mathrm{N}-(\mathrm{C}=\mathrm{O})-\mathrm{C}$ amide groups and the aromatic ring (Figure $\mathrm{S} 7)$. Distances D1 - D4 reflect the orange and green densities in Figure 9C. A notable number of distances D1 - D3 below $4 \AA$ can be found, highlighting potential interactions between the side chains of the pMeOx and the aromatic ring of pPheOzi. Overall, distances show distributions similar to the results of our WAXS experiments. The distance to the ring centroid is the lowest for the pMeOx methyl group, while the backbone is situated further away. These measurements may indicate possible hydrophobic effects between aromatic groups of pPheOzi and the methyl sidechain group of pMeOx. With regard to the pMeOx carbonyl groups, $\mathrm{n}_{\mathrm{Am}} \bullet \bullet \pi^{*}{ }_{\mathrm{Ar}}$ or $\pi_{\mathrm{Am}} \bullet \bullet \bullet \pi_{\mathrm{Ar}}$ interactions may be hypothesized. While it should be mentioned that the existence of specific interactions between lone pairs and aromatic systems was recently challenged ${ }^{63}$ and that the ability of classical force fields for capturing these can 
certainly be questioned, occurrences of an angle $\omega \leq 90^{\circ}$ in combination with a distance lower than $3.8 \AA$ of the carbonyl oxygen to the ring centroid are in accordance with previously published measurements for potential $\mathrm{n}_{\mathrm{Am}} \bullet \bullet \bullet \pi^{*}{ }_{\text {Ar }}$ interactions, e.g. in peptoids. ${ }^{61,64}$ We retrieved very low values for $\omega$ with a median of about $19^{\circ}$ with the above mentioned distance cutoff, indicating suitable conformations for $\mathrm{n}_{\mathrm{Am}} \bullet \bullet \bullet \pi^{*}{ }_{\mathrm{Ar}}$ or $\pi_{\mathrm{Am}} \bullet \bullet \pi_{\mathrm{Ar}}$ interactions. Exemplary simulation snapshots which overlap with the described densities illustrate the interactions between pMeOx and pPheOzi side chains (Figure 9D). While some pMeOx carbonyl groups showed conformations perpendicular to the ring plane (example D-1), in most cases the amide moiety is placed nearly parallel to the aromatic pPheOzi ring (example D-2).

In summary, the MD simulation conclusively corroborates our extensive analytical data, in particular by WAXS and NMR spectroscopy and the formulated mechanism of the hydrophilic pMeOx interacting with the hydrophobic pPheOzi. This results in a notable condensation of the hydrophilic corona and, thus, enables the worm-to-sphere transition upon cooling. This process may be driven by pMeOx side chains mainly interacting with aromatic systems of the pPheOzi blocks via hydrophobic, as well as possible $\pi_{\mathrm{Am}} \bullet \bullet \bullet \pi_{\mathrm{Ar}}$ and $\mathrm{n}_{\mathrm{Am}} \bullet \bullet \bullet \pi^{*}$ Ar interactions.

\section{Conclusion}

Using a wide selection of complementary analytical tools, we gained a detailed picture of molecular interactions responsible for an unusual order-order transition in conjunction with an inverse thermogelation of aqueous solutions of $\mathrm{pMeOx}_{35}-b-\mathrm{pPheOzi}_{15}-b-\mathrm{pMeOx_{35 }}$. DLS and SAXS analysis confirmed the previously described reversible worm-to-spheres transition upon heating. Raman spectroscopy, micro-calorimetry, fluorescence spectroscopy and in particular detailed NMR spectroscopic studies at different temperatures revealed novel and specific polymer/polymer interactions between the hydrophilic pMeOx blocks and the hydrophobic aromatic pPheOzi moieties in the hydrogel state. Comparison with similar, tertiary amide containing systems in the literature and in silico molecular dynamics modeling led us to propose hydrophobic, $n \bullet \bullet \bullet \pi^{*}{ }_{\mathrm{Ar}}$ and/or more pronounced $\pi \bullet \bullet \pi_{\mathrm{Ar}}$ interactions between the carbonyl moieties in the hydrophilic pMeOx block and 
the aromatic rings in the hydrophobic pPheOzi block to be responsible for the order-order transition and inverse thermogelation. To the best of our knowledge, the described system is not only the first example of a sphere-to-worm order-order transition that leads to inverse thermogelling, it is also the first example in which specific interactions on molecular level are critically affecting the self-assembly in synthetic polymer amphiphiles. This mechanistic elucidation should allow optimization and tuning of this unusual system by structural variations, e.g. in the aromatic ring system, but also opens new avenues to design smart materials.

\section{Notes}

$\mathrm{RL}$ and $\mathrm{HL}$ are listed as inventors on a patent application pertinent to some materials in the present work. The other authors declare no competing financial interest.

\section{Acknowledgment}

The authors would like to gratefully acknowledge support by the Deutsche Forschungsgemeinschaft (DFG, German Research Foundation) - project number 326998133 - TRR 225 (subproject A03), awarded to R.L. and project number 440955393 awarded to A.-C.P. The authors thank Christian May for technical assistance and Bernhard Schummer for valuable discussions. We are very grateful for the HDRC-Software version 6.3.1 provided by O. Nirschl and K. Fischer, Physical Chemistry of Polymers at the Johannes Gutenberg University Mainz led by Prof. Dr. Sebastian Seiffert (formerly Prof. Dr. Manfred Schmidt) for DLS data analysis. Light scattering experiments were possible through support of the Deutsche Forschungsgemeinschaft (INST 93/774-1 FUGG). T.L. and E.S.L. acknowledge Academy of Finland for funding of the current research (Project Nos. 316893 and 323669). The authors are also grateful to Dr. Alexander Efimov for BPC12 synthesis. We also thank helpful discussions with Heikki Tenhu and the late Françoise Winnik.

\section{References}

(1) Zhang, Q.; Weber, C.; Schubert, U. S.; Hoogenboom, R. Thermoresponsive polymers with lower critical solution temperature: from fundamental aspects and measuring techniques to recommended turbidimetry conditions. Materials Horizons 2017, 4, 109-116.

(2) Seuring, J.; Agarwal, S. Polymers with upper critical solution temperature in aqueous solution. Macromol Rapid Commun 2012, 33, 1898-1920.

(3) Niskanen, J.; Tenhu, H. How to manipulate the upper critical solution temperature (UCST)? Polymer Chemistry 2017, 8, 220-232.

(4) Lorson, T.; Jaksch, S.; Lubtow, M. M.; Jungst, T.; Groll, J.; Luhmann, T.; Luxenhofer, R. A Thermogelling Supramolecular Hydrogel with Sponge-Like Morphology as a Cytocompatible Bioink. Biomacromolecules 2017, 18, 2161-2171.

(5) Hoffman, A. S. Applications of thermally reversible polymers and hydrogels in therapeutics and diagnostics. Journal of Controlled Release 1987, 6, 297-305. 
(6) Klouda, L.; Mikos, A. G. Thermoresponsive hydrogels in biomedical applications. European Journal of Pharmaceutics and Biopharmaceutics 2008, 68, 34-45.

(7) Hecht, E.; Mortensen, K.; Hoffmann, H. L3 Phase in a Binary Block Copolymer/Water System. Macromolecules 1995, 28, 5465-5476.

(8) Lorson, T.; Lübtow, M. M.; Wegener, E.; Haider, M. S.; Borova, S.; Nahm, D.; Jordan, R.; Sokolski-Papkov, M.; Kabanov, A. V.; Luxenhofer, R. Poly(2-oxazoline)s based biomaterials: A comprehensive and critical update. Biomaterials 2018, 178, 204-280.

(9) Guillerm, B.; Monge, S.; Lapinte, V.; Robin, J.-J. How to Modulate the Chemical Structure of Polyoxazolines by Appropriate Functionalization. Macromolecular Rapid Communications 2012, 33, 1600-1612.

(10) Sedlacek, O.; Hoogenboom, R. Drug Delivery Systems Based on Poly(2-Oxazoline)s and Poly(2-Oxazine)s. Advanced Therapeutics 2019, 3, 1900168.

(11) Zahoranová, A.; Luxenhofer, R. Poly(2-oxazoline)- and Poly(2-oxazine)-Based SelfAssemblies, Polyplexes, and Drug Nanoformulations-An Update. Advanced Healthcare Materials 2021, 10, 2001382.

(12) Park, J.-S.; Akiyama, Y.; Winnik, F. M.; Kataoka, K. Versatile Synthesis of EndFunctionalized Thermosensitive Poly(2-isopropyl-2-oxazolines). Macromolecules 2004, 37, 67866792.

(13) Huber, S.; Hutter, N.; Jordan, R. Effect of end group polarity upon the lower critical solution temperature of poly(2-isopropyl-2-oxazoline). Colloid and Polymer Science 2008, 286, 16531661.

(14) Obeid, R.; Maltseva, E.; Thünemann, A. F.; Tanaka, F.; Winnik, F. M. Temperature Response of Self-Assembled Micelles of Telechelic Hydrophobically Modified Poly(2-alkyl-2oxazoline)s in Water. Macromolecules 2009, 42, 2204-2214.

(15) Obeid, R.; Tanaka, F.; Winnik, F. M. Heat-Induced Phase Transition and Crystallization of Hydrophobically End-Capped Poly(2-isopropyl-2-oxazoline)s in Water. Macromolecules 2009, 42, 5818-5828.

(16) Monnery, B. D.; Hoogenboom, R. Thermoresponsive hydrogels formed by poly(2oxazoline) triblock copolymers. Polymer Chemistry 2019, 10, 3480-3487.

(17) Lübtow, M. M.; Mrlik, M.; Hahn, L.; Altmann, A.; Beudert, M.; Lühmann, T.; Luxenhofer, R. Temperature-Dependent Rheological and Viscoelastic Investigation of a Poly(2methyl-2-oxazoline)-b-poly(2-iso-butyl-2-oxazoline)-b-poly(2-methyl-2-oxazoline)-Based Thermogelling Hydrogel. 2019, 10, 36.

(18) Blanazs, A.; Verber, R.; Mykhaylyk, O. O.; Ryan, A. J.; Heath, J. Z.; Douglas, C. W. I.; Armes, S. P. Sterilizable Gels from Thermoresponsive Block Copolymer Worms. Journal of the American Chemical Society 2012, 134, 9741-9748.

(19) Cunningham, V. J.; Ratcliffe, L. P. D.; Blanazs, A.; Warren, N. J.; Smith, A. J.; Mykhaylyk, O. O.; Armes, S. P. Tuning the critical gelation temperature of thermo-responsive diblock copolymer worm gels. Polymer Chemistry 2014, 5, 6307-6317.

(20) Penfold, N. J. W.; Lovett, J. R.; Verstraete, P.; Smets, J.; Armes, S. P. Stimulusresponsive non-ionic diblock copolymers: protonation of a tertiary amine end-group induces vesicleto-worm or vesicle-to-sphere transitions. Polymer Chemistry 2017, 8, 272-282.

(21) Ratcliffe, L. P. D.; Derry, M. J.; laniro, A.; Tuinier, R.; Armes, S. P. A Single Thermoresponsive Diblock Copolymer Can Form Spheres, Worms or Vesicles in Aqueous Solution. Angew Chem Int Ed Engl 2019, 58, 18964-18970.

(22) Hahn, L.; Maier, M.; Stahlhut, P.; Beudert, M.; Flegler, V.; Forster, S.; Altmann, A.; Toppke, F.; Fischer, K.; Seiffert, S.; Bottcher, B.; Luhmann, T.; Luxenhofer, R. Inverse Thermogelation of Aqueous Triblock Copolymer Solutions into Macroporous Shear-Thinning 3D Printable Inks. ACS Appl Mater Interfaces 2020, 12, 12445-12456.

(23) Hahn, L.; Lübtow, M. M.; Lorson, T.; Schmitt, F.; Appelt-Menzel, A.; Schobert, R.; Luxenhofer, R. Investigating the Influence of Aromatic Moieties on the Formulation of Hydrophobic Natural Products and Drugs in Poly(2-oxazoline)-Based Amphiphiles. Biomacromolecules 2018, 19, 3119-3128. 
(24) Dreiss, C. A.; Jack, K. S.; Parker, A. P. On the absolute calibration of bench-top smallangle X-ray scattering instruments: a comparison of different standard methods. Journal of Applied Crystallography 2006, 39, 32-38.

(25) Zhang, F.; llavsky, J.; Long, G. G.; Quintana, J. P. G.; Allen, A. J.; Jemian, P. R. Glassy Carbon as an Absolute Intensity Calibration Standard for Small-Angle Scattering. Metallurgical and Materials Transactions A 2010, 41, 1151-1158.

(26) Pauw, B. R.; Smith, A. J.; Snow, T.; Terrill, N. J.; Thunemann, A. F. The modular smallangle X-ray scattering data correction sequence. Journal of Applied Crystallography 2017, 50, 18001811.

(27) Konefał, R.; Spěváček, J.; Černoch, P. Thermoresponsive poly(2-oxazoline) homopolymers and copolymers in aqueous solutions studied by NMR spectroscopy and dynamic light scattering. European Polymer Journal 2018, 100, 241-252.

(28) Choe, C.; Lademann, J.; Darvin, M. E. Depth profiles of hydrogen bound water molecule types and their relation to lipid and protein interaction in the human stratum corneum in vivo. Analyst 2016, 141, 6329-6337.

(29) Unal, M.; Akkus, O. Shortwave-infrared Raman spectroscopic classification of water fractions in articular cartilage ex vivo. Journal of biomedical optics 2018, 23, 1-11.

(30) Levitt, J. A.; Kuimova, M. K.; Yahioglu, G.; Chung, P.-H.; Suhling, K.; Phillips, D. Membrane-Bound Molecular Rotors Measure Viscosity in Live Cells via Fluorescence Lifetime Imaging. The Journal of Physical Chemistry C 2009, 113, 11634-11642.

(31) Lisitsyna, E.; Efimov, A.; Depresle, C.; Cauchois, P.; Vuorimaa-Laukkanen, E.; Laaksonen, T.; Durandin, N. Deciphering Multiple Critical Parameters of Polymeric Self-Assembly by Fluorescence Spectroscopy of a Single Molecular Rotor BODIPY-C12. Macromolecules 2021, 54, 655664.

(32) Dent, M. R.; López-Duarte, I.; Dickson, C. J.; Geoghegan, N. D.; Cooper, J. M.; Gould, I. R.; Krams, R.; Bull, J. A.; Brooks, N. J.; Kuimova, M. K. Imaging phase separation in model lipid membranes through the use of BODIPY based molecular rotors. Physical Chemistry Chemical Physics 2015, 17, 18393-18402.

(33) Bond, S. D.; Leimkuhler, B. J.; Laird, B. B. The Nosé-Poincaré Method for Constant Temperature Molecular Dynamics. Journal of Computational Physics 1999, 151, 114-134.

(34) Sturgeon, J. B.; Laird, B. B. Symplectic algorithm for constant-pressure molecular dynamics using a Nosé-Poincaré thermostat. The Journal of Chemical Physics 2000, 112, 3474-3482.

(35) Maier, J. A.; Martinez, C.; Kasavajhala, K.; Wickstrom, L.; Hauser, K. E.; Simmerling, C. ff14SB: Improving the Accuracy of Protein Side Chain and Backbone Parameters from ff99SB. Journal of Chemical Theory and Computation 2015, 11, 3696-3713.

(36) Gerber, P. R.; Müller, K. MAB, a generally applicable molecular force field for structure modelling in medicinal chemistry. Journal of computer-aided molecular design 1995, 9, 251268.

(37) Labute, P. The generalized Born/volume integral implicit solvent model: Estimation of the free energy of hydration using London dispersion instead of atomic surface area. $J$ Comput Chem 2008, 29, 1693-1698.

(38) MOE. Molecular Operating Environment 2019.01. (Chemical Computing Group ULC, 1010 Sherbrooke St. West, Suite \#910, Montreal, QC, Canada, H3A 2R7. 2019.

(39) Yan, H.; Han, Z.; Li, K.; Li, G.; Wei, X. Molecular Dynamics Simulation of the pHInduced Structural Transitions in CTAB/NaSal Solution. Langmuir 2018, 34, 351-358.

(40) Wang, Z.; Larson, R. G. Molecular Dynamics Simulations of Threadlike Cetyltrimethylammonium Chloride Micelles: Effects of Sodium Chloride and Sodium Salicylate Salts. The Journal of Physical Chemistry B 2009, 113, 13697-13710.

(41) Wiest, J.; Kehrein, J.; Saedtler, M.; Schilling, K.; Cataldi, E.; Sotriffer, C. A.; Holzgrabe, U.; Rasmussen, T.; Böttcher, B.; Cronin-Golomb, M.; Lehmann, M.; Jung, N.; Windbergs, M.; Meinel, L. Controlling Supramolecular Structures of Drugs by Light. Molecular Pharmaceutics 2020, 17, 47044708. 
(42) Bayly, C. I.; Cieplak, P.; Cornell, W.; Kollman, P. A. A well-behaved electrostatic potential based method using charge restraints for deriving atomic charges: the RESP model. The Journal of Physical Chemistry 1993, 97, 10269-10280.

(43) Frisch, M. J.; Trucks, G. W.; Schlegel, H. B.; Scuseria, G. E.; Robb, M. A.; Cheeseman, J. R.; Scalmani, G.; Barone, V.; Petersson, G. A.; Nakatsuji, H.; Li, X.; Caricato, M.; Marenich, A. V.; Bloino, J.; Janesko, B. G.; Gomperts, R.; Mennucci, B.; Hratchian, H. P.; Ortiz, J. V.; Izmaylov, A. F.; Sonnenberg, J. L.; Williams; Ding, F.; Lipparini, F.; Egidi, F.; Goings, J.; Peng, B.; Petrone, A.; Henderson, T.; Ranasinghe, D.; Zakrzewski, V. G.; Gao, J.; Rega, N.; Zheng, G.; Liang, W.; Hada, M.; Ehara, M.; Toyota, K.; Fukuda, R.; Hasegawa, J.; Ishida, M.; Nakajima, T.; Honda, Y.; Kitao, O.; Nakai, H.; Vreven, T.; Throssell, K.; Montgomery Jr., J. A.; Peralta, J. E.; Ogliaro, F.; Bearpark, M. J.; Heyd, J. J.; Brothers, E. N.; Kudin, K. N.; Staroverov, V. N.; Keith, T. A.; Kobayashi, R.; Normand, J.; Raghavachari, K.; Rendell, A. P.; Burant, J. C.; Iyengar, S. S.; Tomasi, J.; Cossi, M.; Millam, J. M.; Klene, M.; Adamo, C.; Cammi, R.; Ochterski, J. W.; Martin, R. L.; Morokuma, K.; Farkas, O.; Foresman, J. B.; Fox, D. J.: Gaussian 16 Rev. C.01. Wallingford, CT, 2016.

(44) Wang, J.; Wolf, R. M.; Caldwell, J. W.; Kollman, P. A.; Case, D. A. Development and testing of a general amber force field. J Comput Chem 2004, 25, 1157-1174.

(45) Case, D. A.; Cheatham, T. E., 3rd; Darden, T.; Gohlke, H.; Luo, R.; Merz, K. M., Jr.; Onufriev, A.; Simmerling, C.; Wang, B.; Woods, R. J. The Amber biomolecular simulation programs. J Comput Chem 2005, 26, 1668-1688.

(46) Jorgensen, W. L.; Chandrasekhar, J.; Madura, J. D.; Impey, R. W.; Klein, M. L. Comparison of simple potential functions for simulating liquid water. 1983, 79, 926-935.

(47) Phillips, J. C.; Braun, R.; Wang, W.; Gumbart, J.; Tajkhorshid, E.; Villa, E.; Chipot, C.; Skeel, R. D.; Kalé, L.; Schulten, K. Scalable molecular dynamics with NAMD. J Comput Chem 2005, 26, 1781-1802.

(48) Darden, T.; York, D.; Pedersen, L. Particle mesh Ewald: An N. $\log (\mathrm{N})$ method for Ewald sums in large systems. 1993, 98, 10089-10092.

(49) Roe, D. R.; Cheatham, T. E. PTRAJ and CPPTRAJ: Software for Processing and Analysis of Molecular Dynamics Trajectory Data. Journal of Chemical Theory and Computation 2013, 9, 30843095.

(50) Humphrey, W.; Dalke, A.; Schulten, K. VMD: visual molecular dynamics. Journal of molecular graphics 1996, 14, 33-38, 27-38.

(51) Renouprez, A. J.: Small-Angle X-Ray Scattering. In Catalyst Characterization: Physical Techniques for Solid Materials; Imelik, B., Vedrine, J. C., Eds.; Springer US: Boston, MA, 1994; pp 445465.

(52) Hyland, L. L.; Taraban, M. B.; Yu, Y. B. Using Small-Angle Scattering Techniques to Understand Mechanical Properties of Biopolymer-Based Biomaterials. Soft Matter 2013, 9, 10.1039/C1033SM51209F.

(53) Baral, A.; Basak, S.; Basu, K.; Dehsorkhi, A.; Hamley, I. W.; Banerjee, A. Timedependent gel to gel transformation of a peptide based supramolecular gelator. Soft Matter 2015, $11,4944-4951$.

(54) Kruszynski, R.; Sierański, T. Can Stacking Interactions Exist Beyond the Commonly Accepted Limits? Crystal Growth \& Design 2016, 16, 587-595.

(55) Vyšniauskas, A.; López-Duarte, I.; Duchemin, N.; Vu, T.-T.; Wu, Y.; Budynina, E. M.; Volkova, Y. A.; Peña Cabrera, E.; Ramírez-Ornelas, D. E.; Kuimova, M. K. Exploring viscosity, polarity and temperature sensitivity of BODIPY-based molecular rotors. Physical Chemistry Chemical Physics 2017, 19, 25252-25259.

(56) Boireau-Adamezyk, E.; Baillet-Guffroy, A.; Stamatas, G. N. Mobility of Water Molecules in the Stratum Corneum: Effects of Age and Chronic Exposure to the Environment. Journal of Investigative Dermatology 2014, 134, 2046-2049.

(57) Vyumvuhore, R.; Tfayli, A.; Duplan, H.; Delalleau, A.; Manfait, M.; Baillet-Guffroy, A. Effects of atmospheric relative humidity on Stratum Corneum structure at the molecular level: ex vivo Raman spectroscopy analysis. Analyst 2013, 138, 4103-4111. 
(58) Hiller, W.; Engelhardt, N.; Kampmann, A.-L.; Degen, P.; Weberskirch, R. Micellization and Mobility of Amphiphilic Poly(2-oxazoline) Based Block Copolymers Characterized by $1 \mathrm{H}$ NMR Spectroscopy. Macromolecules 2015, 48, 4032-4045.

(59) Brown, S. P. Advanced solid-state NMR methods for characterising structure and selfassembly in supramolecular chemistry, polymers and hydrogels. Current Opinion in Colloid \& Interface Science 2018, 33, 86-98.

(60) Newberry, R. W.; Raines, R. T. The $n \rightarrow \pi^{*}$ Interaction. Accounts of Chemical Research 2017, 50, 1838-1846.

(61) Gorske, B. C.; Bastian, B. L.; Geske, G. D.; Blackwell, H. E. Local and Tunable $n \rightarrow \pi^{*}$ Interactions Regulate Amide Isomerism in the Peptoid Backbone. Journal of the American Chemical Society 2007, 129, 8928-8929.

(62) Thurn, H.; Hoffmann, H. Evidence of Sticky Contacts between Wormlike Micelles in Viscoelastic Surfactant Solutions. Langmuir 2019, 35, 12192-12204.

(63) Jia, C.; Miao, H.; Hay, B. P. Crystal Structure Evidence for the Directionality of Lone Pair- $\pi$ interactions: Fact or Fiction? Crystal Growth \& Design 2019, 19, 6806-6821.

(64) Egli, M.; Sarkhel, S. Lone Pair-Aromatic Interactions: To Stabilize or Not to Stabilize. Accounts of Chemical Research 2007, 40, 197-205. 